\title{
Estudio sobre el contenido óptimo de yeso en los cementos, su influencia en las resistencias iniciales
}

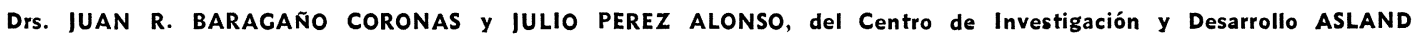

\section{N T R O D U C C I O N}

El tema del contenido óptimo de yeso en los cementos está ampliamente investigado, encontrándose estudios sobre el mismo tanto en los textos clásicos sobre cementos, Bogue (1), Taylor (2), Keil (3), como en revistas más o menos especializadas: Revue de Matériaux de Construction (4) (5) (6) (7), Zement-Kalk-Gips (8), Ultimos Avances (9) (10) (11), así como en los trabajos presentados en los distintos Congresos de la Química del Cemento, Whashington (12), Tokio (13) y Moscou (14).

La A.S.T.M. tiene publicado sobre el mismo tema la designación: C 563-72.

En la bibliografía se indican desde fórmulas matemáticas, como en el caso de LERCH (15), que propone que la cantidad de yeso mínimo a adicionar en un cemento venga dictada por la siguente expresión

$$
\mathrm{SO}_{3}=0,092\left(\mathrm{AC}_{3}\right)+1,71\left(\mathrm{Na}_{2} \mathrm{O}\right)+0,94\left(\mathrm{~K}_{2} \mathrm{O}\right)+1,23
$$

hasta los factores más variados que pueden influir en la velocidad de hidratación de los cementos (contenidos en $\mathrm{SC}_{3}, \mathrm{SC}_{2}, \mathrm{AC}_{3}, \mathrm{AFC}_{4}$, álcalis, cal libre, finura, enfriamiento del clínker, temperatura y tiempo de ensilado, así como la relación agua/cemento empleado en el amasado y la temperatura de curado entre otras); sin embargo, se llega a la conclusión que cada clínker es distinto y necesita un porcentaje ideal de yeso que hay que determinar para obtener las máximas resistencias mecánicas a corto plazo, la mínima expansión sumergido en agua y la más baja retracción de la pasta al aire.

Por otra parte, no todos los cementos son destinados para el mismo fin y su contenido en $\mathrm{SO}_{3}$ debe estar en función de su aplicación y entorno.

Sin profundizar en detalle de las distintas teorías existentes, lo que sí parece claro es que depende, principalmente, de:

a) Contenido de aluminato tricálcico.

b) Contenido en álcalis.

c) Finura del cemento.

Teniendo en cuenta todas estas premisas y su fin, hemos tratado de encauzar este estudio bajo los siguientes aspectos:

1. ${ }^{\circ}$ Ensayo a escala de laboratorio.

2. Ensayo industrial basado en los datos obtenidos anteriormente.

3. Análisis estadístico de los cementos obtenidos industrialmente. 


\section{ENSAYO A ESCALA DE LABORATORIO}

\section{RESULTADO DE LOS ENSAYOS A ESCALA DE LABORATORIO}

Para nuestros ensayos hemos partido de: clínker de hornos Lépol con alto contenido en $\mathrm{SO}_{3}$ y álcalis, y clínker de hornos Allis-Chalmers de tiro abierto y, por ello, con mayores volatilizaciones de los compuestos alcalinos. (Fca. n. ${ }^{\circ} 3$ ).

De cada tipo de clínker se hicieron tres moliendas con distintas superficies Blaine: 2.500, 3.500 y $4.500 \mathrm{~cm}^{2} / \mathrm{g}$, esto es, las alcanzadas en los cementos puzolánico y P-350, P-450 y P-450 ARI, respectivamente.

En cada serie se añadió yeso con un contenido en $\mathrm{SO}_{4} \mathrm{Ca} \cdot 2 \mathrm{H}_{2} \mathrm{O}$ de $97,4 \%$ equivalente a un $45,3 \%$ de $\mathrm{SO}_{3}$ molido a un rechazo de 10,0 sobre 4.900 mallas $/ \mathrm{cm}^{2}$ y en contenidos que van de 1,5 a $7,0 \%$ que, junto al $\mathrm{SO}_{3}$ del clínker, hace que los doce tipos de cemento formados tengan un porcentaje de $\mathrm{SO}_{3}$ desde un mínimo de $1,2 \%$ a un máximo cercano al $4 \%$.

Ante la imposibilidad de realizar todos los ensayos a la vez y con el fin de evitar al máximo los errores, se ensayaron cada serie de doce cementos en un mismo día para realizar las roturas correspondientes a las 12,16 y 24 horas, y en otro día, las correspondientes a 3,7 y 28 días.

En cada tipo de cemento, además, se determinó el porcentaje total de $\mathrm{SO}_{3}$, finuras, S.E. Blaine, principio y fin de fraguado, expansión al autoclave y retracción a temperatura ambiente hasta la edad de 56 días.

Las roturas se realizaron con una prensa automática MOHR-FEDERHAFF a velocidad constante.

\subsection{Análisis químico de los clínkeres empleados}

\begin{tabular}{|c|c|c|}
\hline & $\begin{array}{c}C \quad L I N K E R \\
\text { Allis-Chalmers }\end{array}$ & Lépol \\
\hline$\%$ RFH & 0.2 & 0,2 \\
\hline$\% \mathrm{SiOO}_{2}$ & 22,6 & 21,9 \\
\hline$\% \quad \mathrm{Al}_{2} \mathrm{O}_{3}$ & 5,2 & 5,3 \\
\hline$\% \mathrm{Fe}_{2} \mathrm{O}_{3}$ & 2,4 & 2,4 \\
\hline$\% \mathrm{CO}_{2}$ & 643 & 64,4 \\
\hline$\% \mathrm{MgO}$ & 2,7 & 2,6 \\
\hline$\% \mathrm{SO}_{3}$ & 0,6 & 0,9 \\
\hline$\% \quad \mathrm{~K}_{2} \mathrm{O}$ & 0,8 & 1,2 \\
\hline$\% \quad \mathrm{Na}_{2} \mathrm{O}$ & 0,3 & 0,4 \\
\hline \% P.F. & 0,6 & 0,7 \\
\hline$\% \mathrm{CaO}$ libre & 1,5 & 1,1 \\
\hline G.S.P. & 90,6 & 93,2 \\
\hline G.S.R. & 88,5 & 91,6 \\
\hline M.H. & 2,1 & 2,2 \\
\hline M.S. & 3,0 & 2,8 \\
\hline M.F. & 2,2 & 2,2 \\
\hline$\% \mathrm{SC}_{3}\left({ }^{*}\right)$ & 45,5 & 52,2 \\
\hline$\% \mathrm{SC}_{2}$ & 30,6 & 23,5 \\
\hline$\% \quad \mathrm{AC}_{3}$ & 9,7 & 10,0 \\
\hline$\% \mathrm{AFC}_{+}$ & 7,3 & 7,3 \\
\hline$\% \quad \mathrm{SC}_{3} / \mathrm{SC}_{2}$ & 1,5 & 2,2 \\
\hline
\end{tabular}

(*) Obtenido según Bogue. 


\subsection{Datos alcanzados con clínker de hornos Allis-Chalmers}

Se exponen a continuación, detallados en las tablas 1.2.1., 1.2.2. y 1.2.3., los valores logrados con clínkeres de hornos Allis-Chalmers al molerlos en un molino de bolas de laboratorio con los distintos porcentajes de yeso $(1,5-7,0)$.

En los gráficos 1.2.1., 1.2.2. y 1.2.3. se representan dichos valores limitando por líneas verticales los considerados como óptimos dentro de los márgenes de error que se suelen cometer en esta clase de trabajos.

T A B L A 1.2 .1$.

Clínker Hornos Allis-Chalmers S.E.B. $\simeq 2.500$

\begin{tabular}{|cccc|}
\hline \multicolumn{4}{c|}{ FINURA } \\
\hline $\mathbf{9 0 0}$ & $\mathbf{4 . 9 0 0}$ & $\mathbf{1 0 . 0 0 0}$ & S.E.B. \\
\hline 1,2 & 9,0 & 18,0 & 2.886 \\
\hline
\end{tabular}

\begin{tabular}{|c|c|c|c|c|c|c|c|c|c|c|c|c|}
\hline \multirow{2}{*}{$\begin{array}{c}\% \\
\text { Yeso } \\
\text { añadido } \\
\end{array}$} & \multirow{2}{*}{$\begin{array}{c}\% \\
\mathrm{SO}_{3} \\
\text { total }\end{array}$} & \multirow[b]{2}{*}{$\begin{array}{l}\text { S.E.B. } \\
\mathrm{cm}^{2} / \mathrm{g}\end{array}$} & \multicolumn{3}{|c|}{ FRACUADO } & \multirow{2}{*}{$\begin{array}{c}\% \\
\text { Expans. } \\
\text { Autọcl. }\end{array}$} & \multicolumn{6}{|c|}{ RESISTENCIAS MECANICAS } \\
\hline & & & Agua & Inicial & Final & & $12 \mathrm{~h}$ & $16 \mathrm{~h}$ & $24 \mathrm{~h}$ & 3 días & 7 días & 28 días \\
\hline 1,5 & 1,4 & 2.979 & 27 & $\mathrm{Oh} 35 \mathrm{~min}$ & $1 \mathrm{~h} 10 \mathrm{~min}$ & 0,08 & $5 / 17$ & $7 / 23$ & $15 / 56$ & $32 / 130$ & $47 / 213$ & $61 / 335$ \\
\hline 2,0 & 1,6 & 3.055 & 27 & I $\mathrm{h} 00 \mathrm{~min}$ & $1 \mathrm{~h} 45 \mathrm{~min}$ & 0,08 & $7 / 21$ & $9 / 28$ & $14 / 65$ & $38 / 150$ & $55 / 255$ & $64 / 364$ \\
\hline 2,5 & 1,7 & 3.103 & 26 & $1 \mathrm{~h} 00 \mathrm{~min}$ & $1 \mathrm{~h} 45 \mathrm{~min}$ & 0,17 & $6 / 18$ & $9 / 34$ & $14 / 61$ & $37 / 163$ & $51 / 255$ & $67 / 358$ \\
\hline 3,0 & 2,1 & 3.135 & 26 & $1 \mathrm{~h} 10 \mathrm{~min}$ & $1 \mathrm{~h} 55 \mathrm{~min}$ & 0,07 & $9 / 31$ & $11 / 43$ & $18 / 87$ & $40 / 168$ & $60 / 280$ & $67 / 370$ \\
\hline 3,5 & 2,4 & 3.177 & 26 & $1 \mathrm{~h} 10 \mathrm{~min}$ & $1 \mathrm{~h} 55 \mathrm{~min}$ & 0,17 & $12 / 43$ & $14 / 58$ & $20 / 85$ & $43 / 183$ & $58 / 271$ & $72 / 386$ \\
\hline 4,0 & 2,5 & 3.224 & 26 & $1 \mathrm{~h} 15 \mathrm{~min}$ & $2 \mathrm{~h} 05 \mathrm{~min}$ & 0,05 & $14 / 53$ & $15 / 70$ & $21 / 99$ & $47 / 193$ & $57 / 275$ & $71 / 378$ \\
\hline 4,5 & 2,8 & 3.245 & 26 & $1 \mathrm{~h} 15 \mathrm{~min}$ & $2 \mathrm{~h} 10 \mathrm{~min}$ & 0,14 & $17 / 65$ & $18 / 88$ & $28 / 125$ & $43 / 193$ & $56 / 274$ & $78 / 406$ \\
\hline 5,0 & 2,9 & 3.260 & 26 & $1 \mathrm{~h} 30 \mathrm{~min}$ & $2 \mathrm{~h} 20 \mathrm{~min}$ & 0,13 & $16 / 64$ & $20 / 87$ & $28 / 12.5$ & $48 / 198$ & $63 / 300$ & $79 / 425$ \\
\hline 5,5 & 3,1 & 3.316 & 26 & $1 \mathrm{~h} 35 \mathrm{~min}$ & $2 \mathrm{~h} 35 \mathrm{~min}$ & 0,13 & $16 / 65$ & $22 / 92$ & $30 / 136$ & $48 / 203$ & $62 / 290$ & $73 / 403$ \\
\hline 6,0 & 3,3 & 3.356 & 26 & $1 \mathrm{~h} 40 \mathrm{~min}$ & $2 \mathrm{~h} 45 \mathrm{~min}$ & 0,12 & $14 / 54$ & $22 / 94$ & $31 / 142$ & $43 / 186$ & $61 / 284$ & $75 / 405$ \\
\hline 6,5 & 3,5 & 3.391 & 26 & $1 \mathrm{~h} 50 \mathrm{~min}$ & $2 \mathrm{~h} 55 \mathrm{~min}$ & 0,10 & $13 / 43$ & $19 / 78$ & $32 / 146$ & $46 / 210$ & $61 / 285$ & $72 / 398$ \\
\hline 7,0 & 3,7 & 3.440 & 26 & $1 \mathrm{~h} 50 \mathrm{~min}$ & $3 \mathrm{~h} 10 \mathrm{~min}$ & 0,08 & $12 / 47$ & $20 / 81$ & $33 / 148$ & $50 / 207$ & $63 / 290$ & $79 / 423$ \\
\hline
\end{tabular}

T A B L A 1.2 .2 .

Clínker Hornos Allis-Chalmers S.E.B. $\simeq 3.500$

\begin{tabular}{|cccc|}
\hline \multicolumn{5}{|c|}{ FINURA } & CLINKER \\
\hline $\mathbf{9 0 0}$ & $\mathbf{4 . 9 0 0}$ & $\mathbf{1 0 . 0 0 0}$ & S.E.B. \\
\hline 1,6 & 5,8 & 11,0 & 3.469 \\
\hline
\end{tabular}

\begin{tabular}{|c|c|c|c|c|c|c|c|c|c|c|c|c|}
\hline $\begin{array}{c}\text { Yeso } \\
\text { añadido }\end{array}$ & $\begin{array}{c}\% \\
\text { SO }_{3} \\
\text { total }\end{array}$ & $\begin{array}{l}\text { S.E.B. } \\
\mathrm{cm}^{2} / \mathrm{g}\end{array}$ & Agua & Inicial & Final & $\begin{array}{c}\% \\
\text { Expans. } \\
\text { Autocl. }\end{array}$ & $12 \mathrm{~h}$ & $16 \mathrm{~h}$ & $24 \mathrm{~h}$ & 3 días & 7 días & 28 días \\
\hline 2,0 & 1,5 & 3.610 & 28 & $1 \mathrm{~h} 10 \mathrm{~min}$ & $1 \mathrm{~h} 40 \mathrm{~min}$ & 0,08 & $7 / 23$ & $10 / 40$ & $18 / 80$ & $39 / 168$ & $54 / 248$ & $67 / 331$ \\
\hline 2,5 & 1,8 & 3.702 & 27 & $1 \mathrm{~h} 10 \mathrm{~min}$ & $1 \mathrm{~h} 45 \mathrm{~min}$ & 0,07 & $7 / 32$ & $11 / 47$ & $18 / 81$ & $42 / 203$ & $56 / 300$ & $69 / 386$ \\
\hline 4,0 & 2,5 & 3.733 & 27 & $1 \mathrm{~h} 20 \mathrm{~min}$ & $2 \mathrm{~h} 10 \mathrm{~min}$ & 0,09 & $17 / 59$ & 18/86 & $25 / 116$ & $50 / 223$ & $61 / 333$ & $74 / 414$ \\
\hline 4,5 & 2,6 & 3.780 & 27 & $1 \mathrm{~h} 25 \mathrm{~min}$ & $2 \mathrm{~h} 10 \mathrm{~min}$ & 0,08 & $17 / 68$ & $20 / 89$ & $28 / 113$ & $50 / 255$ & $65 / 320$ & $71 / 396$ \\
\hline 5,0 & 2,9 & 3.822 & 27 & $1 \mathrm{~h} 30 \mathrm{~min}$ & $2 \mathrm{~h} 20 \mathrm{~min}$ & 0,11 & $18 / 75$ & $22 / 102$ & $28 / 131$ & $52 / 250$ & $63 / 320$ & $78 / 391$ \\
\hline 5,5 & 3,1 & 3.831 & 27 & $1 \mathrm{~h} 30 \mathrm{~min}$ & $2 \mathrm{~h} 20 \mathrm{~min}$ & 0,13 & $19 / 71$ & $21 / 102$ & $30 / 135$ & $53 / 250$ & $60 / 320$ & $78 / 385$ \\
\hline 6,0 & 3,3 & 3.878 & 27 & $1 \mathrm{~h} 40 \mathrm{~min}$ & $2 \mathrm{~h} 40 \mathrm{~min}$ & 0,06 & $19 / 66$ & $24 / 106$ & $30 / 133$ & $57 / 250$ & $65 / 313$ & $83 / 416$ \\
\hline
\end{tabular}


T A B L A 1.2 .3 .

Clínker Hornos Allis-Chalmers S.E.B. $\simeq 4.500$

\begin{tabular}{|c|c|c|c|}
\hline \multicolumn{4}{|c|}{ FINURA CLINKER } \\
\hline 900 & 4.900 & 10.000 & S.E.B. \\
\hline 3,8 & 8,8 & 16,0 & 4.714 \\
\hline
\end{tabular}

\begin{tabular}{|c|c|c|c|c|c|c|c|c|c|c|c|c|}
\hline \multirow{2}{*}{$\begin{array}{c}\% \\
\text { Yeso } \\
\text { añadido }\end{array}$} & \multirow{2}{*}{$\begin{array}{c}\% \\
\mathrm{SO}_{3} \\
\text { total }\end{array}$} & \multirow[b]{2}{*}{$\begin{array}{l}\text { S.E.B. } \\
\mathrm{cm}^{2} / \mathrm{g}\end{array}$} & \multicolumn{3}{|c|}{ FRACUADO } & \multirow{2}{*}{$\begin{array}{c}\% \\
\text { Expans. } \\
\text { Autocl. }\end{array}$} & \multicolumn{6}{|c|}{ RESISTENCIAS MECANICAS } \\
\hline & & & Agua & Inicial & Final & & $12 \mathrm{~h}$ & $16 \mathrm{~h}$ & $24 \mathrm{~h}$ & 3 diạs & 7 días & 28 días \\
\hline 1,5 & 1,3 & 4.802 & 30 & $\mathrm{Oh} 30 \mathrm{~min}$ & $1 \mathrm{~h} 05 \mathrm{~min}$ & 0,06 & $10 / 32$ & $13 / 50$ & $19 / 71$ & $32 / 141$ & $39 / 176$ & $51 / 246$ \\
\hline 2,0 & 1,7 & 4.852 & 30 & $0 \mathrm{~h} 50 \mathrm{~min}$ & $1 \mathrm{~h} 15 \mathrm{~min}$ & 0,06 & $11 / 43$ & $16 / 57$ & $23 / 105$ & $42 / 170$ & $55 / 210$ & $68 / 320$ \\
\hline 2,5 & 1,9 & 4.939 & 29 & $1 \mathrm{~h} 00 \mathrm{~min}$ & $1 \mathrm{~h} 25 \mathrm{~min}$ & 0,04 & $12 / 42$ & $18 / 80$ & $26 / 111$ & $40 / 190$ & $54 / 215$ & $62 / 325$ \\
\hline 3,0 & 2,3 & 5.006 & 29 & $1 \mathrm{~h} 10 \mathrm{~min}$ & $1 \mathrm{~h} 30 \mathrm{~min}$ & 0,04 & $14 / 53$ & $19 / 84$ & $28 / 116$ & $51 / 218$ & $52 / 273$ & $70 / 365$ \\
\hline 3,5 & 2,4 & 5.039 & 29 & $1 \mathrm{~h} 10 \mathrm{~min}$ & $1 \mathrm{~h} 30 \mathrm{~min}$ & 0,00 & $16 / 68$ & $21 / 90$ & $30 / 126$ & $47 / 239$ & $55 / 280$ & $73 / 395$ \\
\hline 4,0 & 2,6 & 5.106 & 29 & $1 \mathrm{~h} 20 \mathrm{~min}$ & $1 \mathrm{~h} 40 \mathrm{~min}$ & 0,03 & $19 / 74$ & $22 / 109$ & $32 / 139$ & $49 / 240$ & $61 / 280$ & $78 / 389$ \\
\hline 45 & 2,9 & 5.154 & 28 & $1 \mathrm{~h} 25 \mathrm{~min}$ & $1 \mathrm{~h} 45 \mathrm{~min}$ & 0,04 & $20 / 81$ & $23 / 112$ & $30 / 145$ & $50 / 243$ & $52 / 268$ & $70 / 375$ \\
\hline 5,0 & 3,0 & 5.219 & 28 & $1 \mathrm{~h} 30 \mathrm{~min}$ & $1 \mathrm{~h} 50 \mathrm{~min}$ & 006 & $21 / 86$ & $26 / 117$ & $32 / 144$ & $53 / 239$ & $56 / 274$ & $70 / 369$ \\
\hline 5,5 & 3,3 & 5.251 & 28 & $1 \mathrm{~h} 30 \mathrm{~min}$ & $1 \mathrm{~h} 55 \mathrm{~min}$ & 0,03 & $20 / 95$ & $27 / 136$ & $33 / 161$ & $46 / 241$ & $62 / 279$ & $68 / 370$ \\
\hline 6,0 & 3,5 & 5.267 & 28 & $1 \mathrm{~h} 35 \mathrm{~m}: \mathrm{n}$ & $2 \mathrm{~h} 10 \mathrm{~min}$ & 0,04 & $22 / 101$ & $30 / 144$ & $36 / 162$ & $49 / 238$ & $57 / 271$ & $74 / 396$ \\
\hline 6,5 & 3,6 & 5.298 & 28 & $1 \mathrm{~h} 35 \mathrm{~min}$ & $2 \mathrm{~h} 10 \mathrm{~min}$ & 0,02 & $22 / 101$ & $29 / 142$ & $37 / 171$ & $52 / 243$ & $62 / 275$ & $78 / 369$ \\
\hline 7,0 & 3,7 & 5.314 & 28 & $1 \mathrm{~h} 40 \mathrm{~min}$ & $2 \mathrm{~h} 30 \mathrm{~min}$ & 0,02 & $23 / 105$ & $29 / 150$ & $36 / 175$ & $49 / 237$ & $59 / 274$ & $70 / 360$ \\
\hline
\end{tabular}

\subsection{Comentario parcial}

Se observa un aumento en la S.E. Blaine de unas 500 unidades al ir aumentando el contenido de yeso de 1,5 a $7,0 \%$, tanto si partimos de un clínker molido a $2.500 \mathrm{~cm}^{2} / \mathrm{g}$ como si es de $4.500 \mathrm{~cm}^{2} / \mathrm{g}$; por tanto un aumento en el contenido del yeso puede falsear el concepto de finura del cemento.

De los tiempos de fraguado, se deduce que el contenido en yeso no debe ser inferior al correspondiente de $1,7 \%$ en $\mathrm{SO}_{3}$, so pena de correr el riesgo de fabricar cementos de fraguados rápidos.

La expansión al autoclave no parece tener relación con el contenido en yeso.

En todos los ensayos hemos trazado líneas verticales indicando el margen en el que se obtienen mejores resistencias, observándose como, a medida que aumenta el tiempo y, por lo general, a partir ya de 3 días, la influencia del porcentaje de yeso no se deja sentir excepto para concentraciones bajas de $\mathrm{SO}_{3}$, con posibilidad de fraguado rápido.

\subsection{Datos alcanzados con el clínker de hornos Lépol}

Al igual que anteriormente, en las tablas 1.4.1., 1.4.2. y 1.4.3. se exponen los valores logrados en los distintos ensayos realizados con un promedio de clínker de hornos Lépol.

En las tablas 1.4.4., 1.4.5. y 1.4.6. se detallan los porcentajes de retracción obtenidos al exponer en el aire, a temperatura ambiente, probetas de pasta pura de $25,4 \times 25,4 \times 287$ milímetros desde un día a ocho semanas.

En los gráficos 1.4.1., 1.4.2., 1.4.3. y 1.4.4. se representan los valores obtenidos en los ensayos mecánicos, así como la retracción alcanzada en el aire a las distintas fechas de medición. 


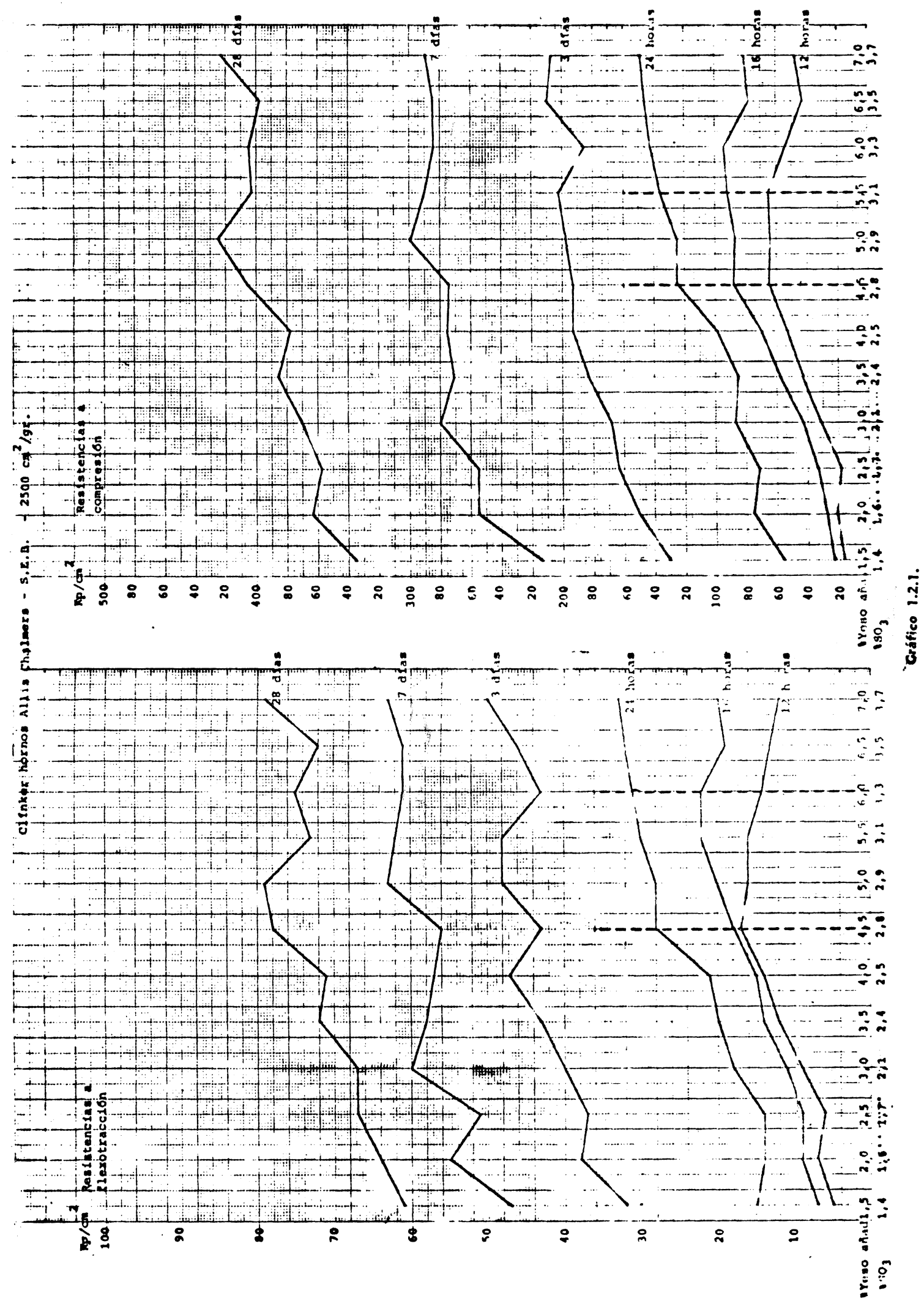




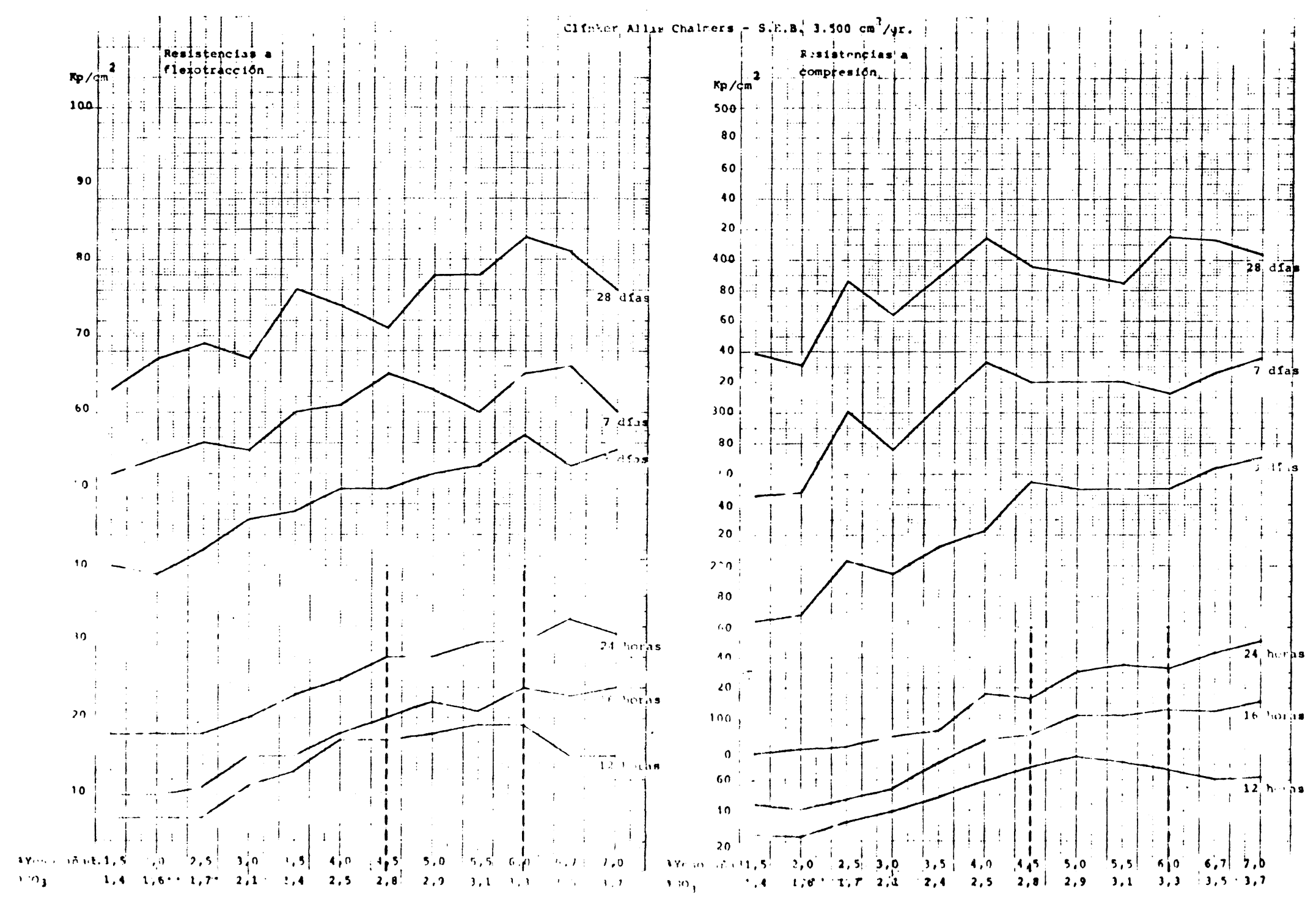

Cráfico 1.2.2. 


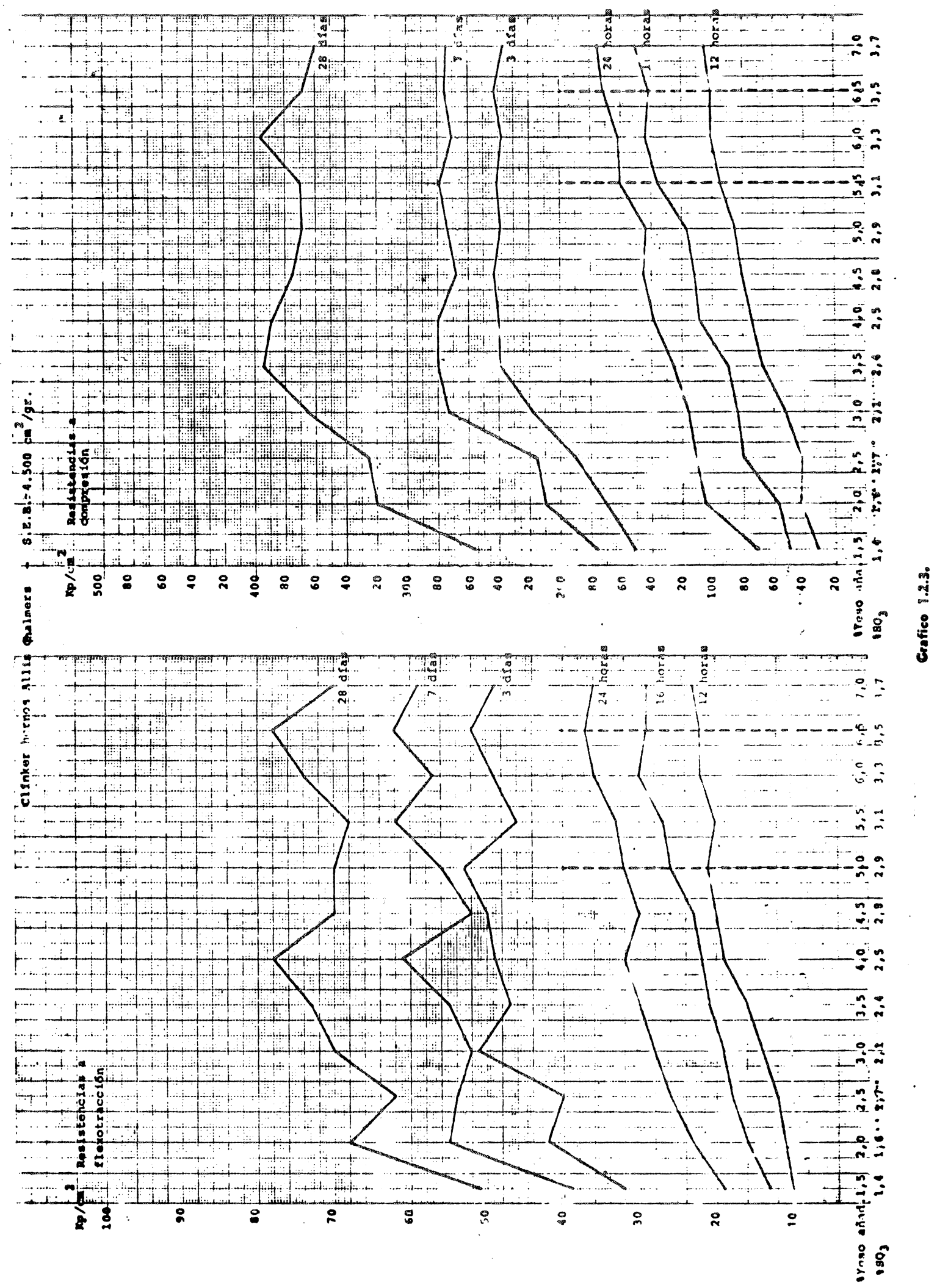


Las verticales indican los valores considerados como óptimos.

T A B L A 1.4 .1$.

Clinker Hornos Lépol S.E.B. $\simeq 2.500$

\begin{tabular}{|cccc|}
\hline \multicolumn{4}{|c|}{ FINURA } \\
\hline 900 & 4.900 & 10.000 & S.E.B. \\
\hline 1.0 & 6.4 & 14,0 & 2.748 \\
\hline
\end{tabular}

\begin{tabular}{|c|c|c|c|c|c|c|c|c|c|c|c|c|c|c|}
\hline \multirow{2}{*}{$\begin{array}{c}\% \\
\text { Yeso } \\
\text { añadido }\end{array}$} & \multirow{2}{*}{$\begin{array}{c}\% \\
\text { so }_{3} \\
\text { total }\end{array}$} & \multirow[b]{2}{*}{$\begin{array}{l}\text { S.E.B. } \\
\mathrm{cm}^{2} / \mathrm{g}\end{array}$} & \multicolumn{4}{|c|}{ FRAGUADO } & \multirow{2}{*}{$\begin{array}{l}\% \\
\text { Expans. } \\
\text { Autocl. }\end{array}$} & \multirow{2}{*}{$\begin{array}{c}\% \text { Retrac. } \\
\text { al aire } \\
56 \text { dias }\end{array}$} & \multicolumn{3}{|c|}{ RESISTENCIAS } & \multicolumn{3}{|c|}{ MECANICAS } \\
\hline & & & Agua & Inicial & & Final & & & $12 \mathrm{~h}$ & $16 \mathrm{~h}$ & $24 \mathrm{~h}$ & 3 días & 7 dias & 28 días \\
\hline 1,5 & 1.3 & 2.977 & 26 & $1 \mathrm{~h} 35 \mathrm{~min}$ & $2 \mathrm{~h}$ & $20 \mathrm{~min}$ & 0.10 & 0,471 & $7 / 34$ & $12 / 50$ & $22 / 88$ & $52 / 209$ & $61 / 288$ & $71 / 365$ \\
\hline 2,0 & 1.5 & 3.038 & 26 & $1 \mathrm{~h} 45 \mathrm{~min}$ & $2 \mathrm{~h}$ & $25 \mathrm{~min}$ & 0.07 & 0.378 & $11 / 40$ & $15 / 60$ & $24 / 98$ & $51 / 234$ & $69 / 309$ & $73 / 418$ \\
\hline 2.5 & 1,6 & 3.034 & 26 & $1 \mathrm{~h} 50 \mathrm{~min}$ & $2 \mathrm{~h}$ & $40 \mathrm{~min}$ & 0.09 & 0.381 & $13 / 50$ & $17 / 79$ & $26 / 116$ & $56 / 231$ & $70 / 309$ & $75 / 406$ \\
\hline 3,0 & 1.9 & 3.061 & 26 & $1 \mathrm{~h} 55 \mathrm{~min}$ & $2 \mathrm{~h}$ & $45 \mathrm{~min}$ & 0.08 & 0,297 & $17 / 55$ & $23 / 102$ & $33 / 158$ & $58 / 255$ & $71 / 339$ & $76 / 42-1$ \\
\hline 3,5 & 2,2 & 3.083 & 26 & $1 \mathrm{~h} 55 \mathrm{~min}$ & $2 \mathrm{~h}$ & $50 \mathrm{~min}$ & 0,05 & 0.293 & $17 / 65$ & $24 / 111$ & $34 / 168$ & $57: 255$ & 72,345 & 8,41, \\
\hline $4, \mathrm{C}$ & 2.6 & 3.173 & 26 & $2 \mathrm{~h} 00 \mathrm{~min}$ & $3 \mathrm{~h}$ & $10 \mathrm{~min}$ & 0,07 & 0.292 & $16 / 61$ & $24 / 110$ & $36 / 169$ & $60 / 275$ & $75 / 349$ & $80 / 439$ \\
\hline 4,5 & 2.8 & 3.143 & 25 & $2 \mathrm{~h} 00 \mathrm{~min}$ & $3 \mathrm{~h}$ & $00 \mathrm{~min}$ & 0,09 & 0,285 & $16 / 60$ & $24 / 111$ & $35 / 184$ & $63 / 279$ & $77 / 346$ & $85 / 448$ \\
\hline 5,0 & 3,0 & 3.196 & 25 & $1 \mathrm{~h} 55 \mathrm{~min}$ & $2 \mathrm{~h}$ & $50 \mathrm{~min}$ & 0,07 & 0.292 & $16 / 58$ & $23 / 95$ & $36 ; 188$ & $64 / 276$ & $79 / 331$ & $86 / 438$ \\
\hline 5,5 & 3,2 & 3.197 & 25 & $2 \mathrm{~h} 00 \mathrm{~min}$ & $3 \mathrm{~h}$ & $10 \mathrm{~min}$ & 0.05 & 0,336 & $12 / 52$ & $22 / 82$ & $36 / 189$ & $62 / 281$ & $74 / 325$ & $79 / 410$ \\
\hline 6,0 & 3,3 & 3.250 & 25 & $2 \mathrm{~h} 00 \mathrm{~min}$ & $3 \mathrm{~h}$ & $10 \mathrm{~min}$ & 0,04 & 0,299 & $13 / 50$ & $19 / 73$ & $30 / 187$ & $58 / 279$ & $75 / 339$ & $77 / 413$ \\
\hline 6,5 & 3.5 & 3.303 & 25 & $2 \mathrm{~h} 50 \mathrm{~min}$ & $3 \mathrm{~h}$ & $10 \mathrm{~min}$ & 0,03 & 0,387 & $12 / 46$ & $19 / 90$ & $28 / 158$ & $66 / 288$ & $79 / 358$ & $85 / 450$ \\
\hline 7,0 & 3,8 & 3.378 & 25 & $2 \mathrm{H}, 10 \mathrm{~min}$ & $3 \mathrm{~h}$ & $20 \mathrm{~min}$ & 0.04 & 0,342 & $12 / 46$ & $18 / 85$ & $27 / 150$ & $63 / 282$ & $78 / 341$ & $84 / 448$ \\
\hline
\end{tabular}

T A в L A 1.4 .2 .

Clinker Hornos Lépol S.E.B. $\simeq 3.500$

\begin{tabular}{|cccc|}
\hline \multicolumn{4}{c}{ FINURA } \\
\hline 900 & 4.900 & 10.000 & S.E.B. \\
\hline 2.2 & 6.0 & 8.0 & 3.450 \\
\hline
\end{tabular}

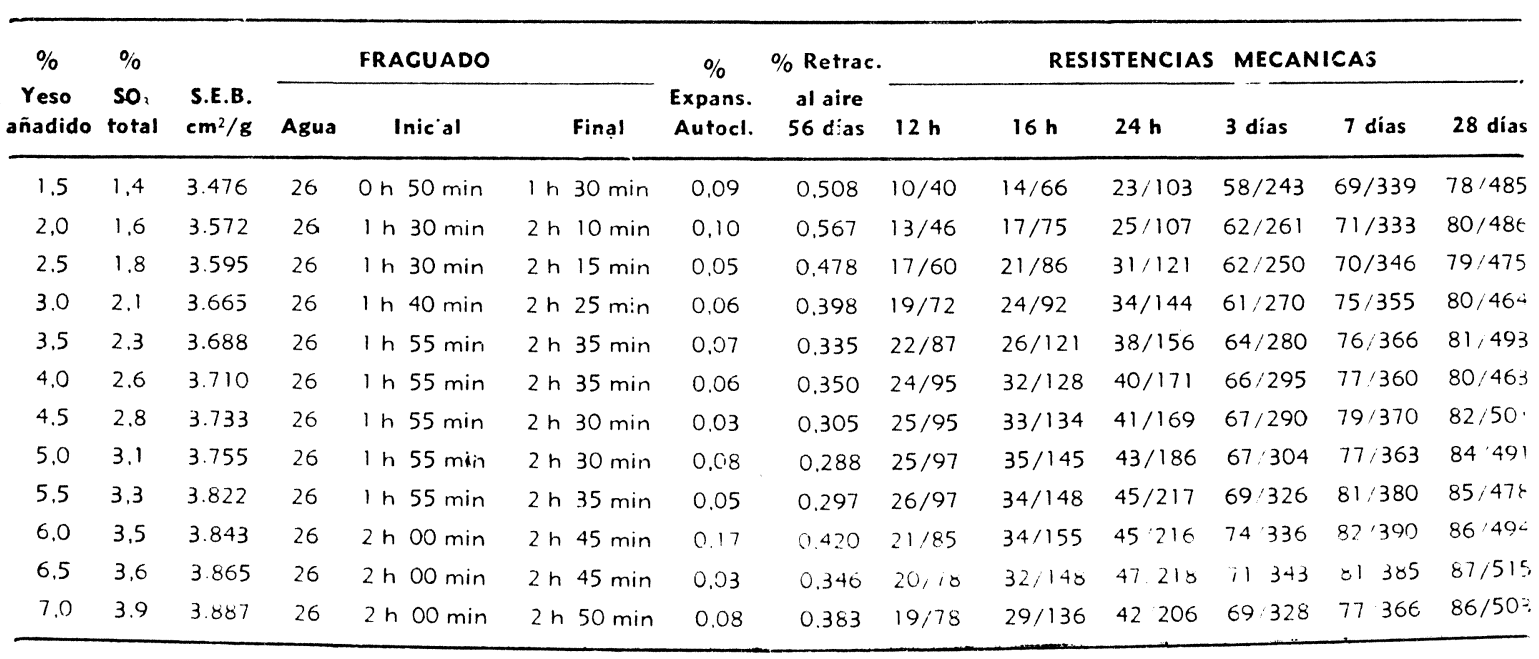


T A в L A 1.4 .3 .

Clínker Hornos Lépol S.E.B. $\simeq 4.500$

\begin{tabular}{|cccc|}
\hline \multicolumn{5}{c|}{ FINURA } & CLINKER \\
\hline 900 & 4.900 & 10.000 & S.E.B. \\
\hline 2,4 & 6,4 & 11,0 & 4520 \\
\hline
\end{tabular}

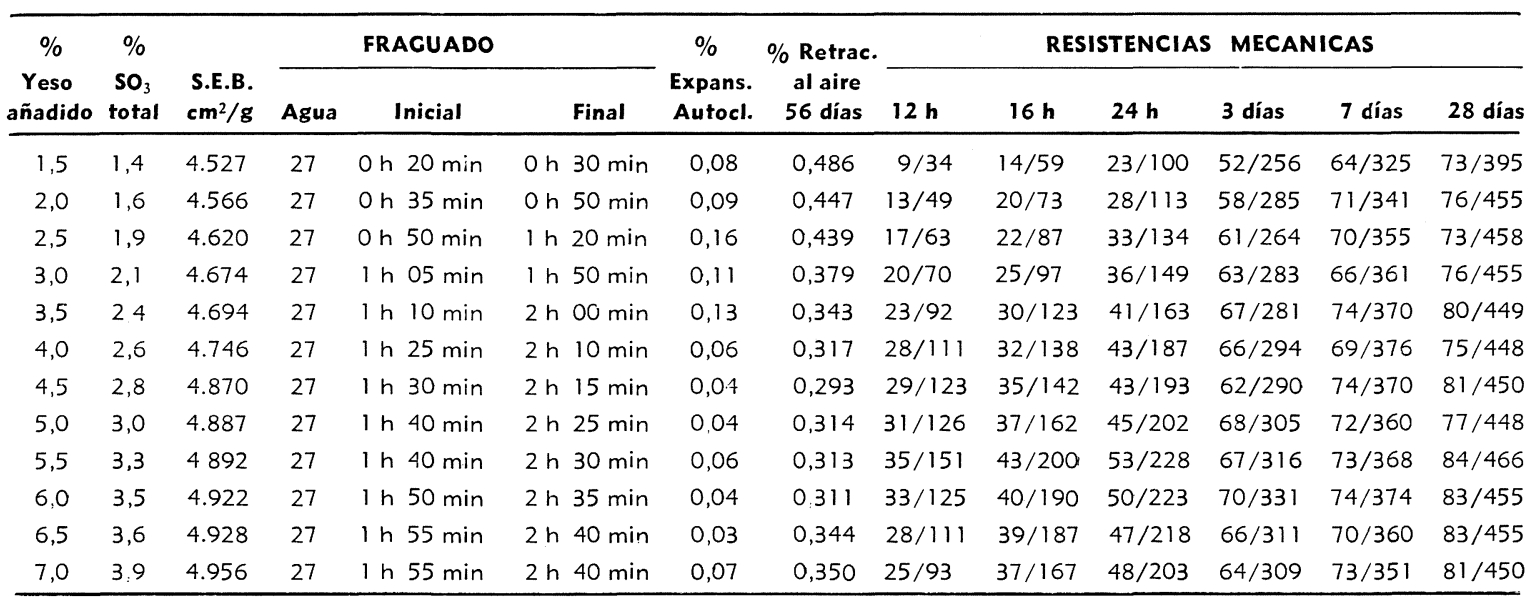

T A B L A 1.4 .4 .

Clínker Hornos Lépol S. Blaine $2.500 \mathrm{~cm}^{2} / \mathrm{g}$

Retracción de pasta pura expuesta al aire a temperatura ambiente

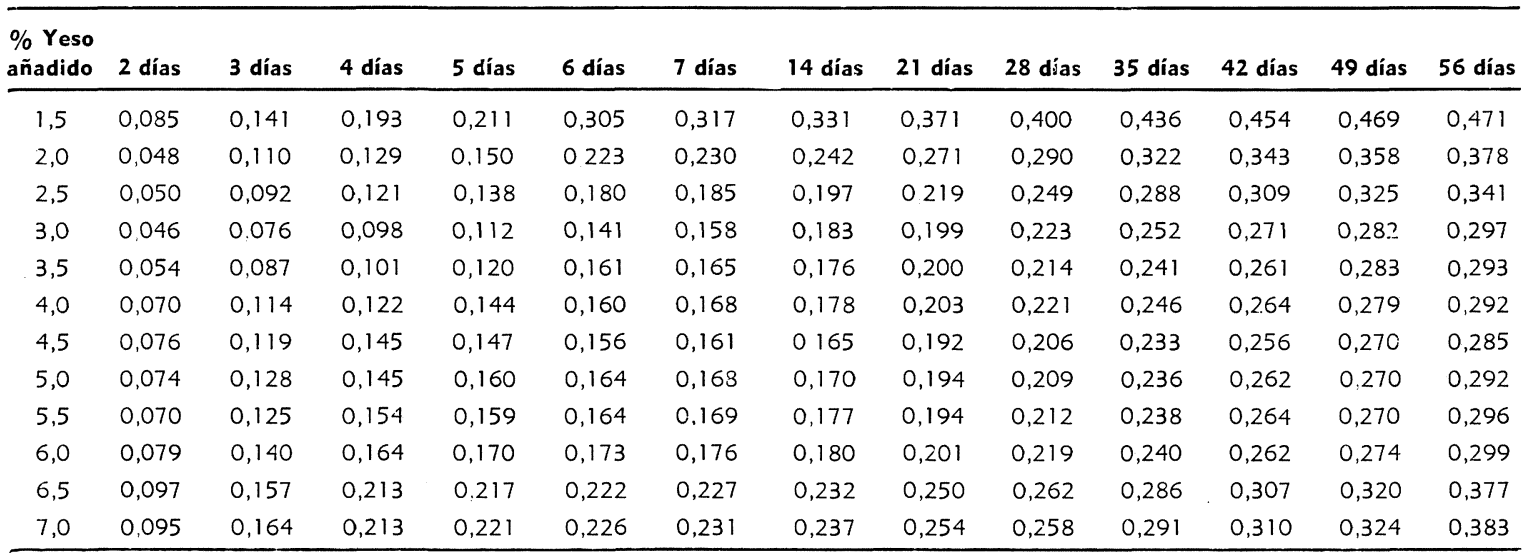

T A в L A 1.4 .5 .

Clínker Hornos Lépol S. Blaine $3.500 \mathrm{~cm}^{2} / g$

Retracción de pasta pura expuesta al aire a temperatura ambiente

\begin{tabular}{|c|c|c|c|c|c|c|c|c|c|c|c|c|c|}
\hline 1,5 & 0,090 & 0,132 & 0,273 & 0,290 & 0,301 & 0,318 & 0,331 & 0,373 & 0,403 & 0,468 & 0,483 & 0,493 & 0,508 \\
\hline 2,5 & 0,076 & 0,106 & 0,186 & $0,192$. & 0,202 & 0,216 & 0,222 & 0,260 & 0,282 & 0,332 & 0,352 & 0,408 & 0,430 \\
\hline 3,0 & 0,068 & 0,089 & 0,149 & 0,158 & 0,168 & 0,176 & 0,181 & 0,218 & 0,245 & 0,290 & 0,311 & 0374 & 0,398 \\
\hline 4,5 & 0,115 & 0,136 & 0,149 & 0,155 & 0,157 & 0,165 & 0,177 & 0,202 & 0,234 & 0,260 & 0,271 & 0,288 & 0,305 \\
\hline 5,0 & 0,132 & 0,139 & 0,150 & 0,159 & 0,163 & 0,169 & 0,176 & 0,202 & 0,228 & 0,256 & 0,264 & 0279 & 0,288 \\
\hline 5,5 & 0,145 & 0,151 & 0,160 & 0,169 & 0,178 & 0,184 & 0,188 & 0,217 & 0,236 & 0,262 & 0,272 & 0,288 & 0,297 \\
\hline 6,0 & 0,160 & 0,166 & 0,170 & 0,182 & 0,187 & 0,191 & 0,200 & 0,232 & 0,256 & 0,285 & 0,293 & 0,305 & 0,320 \\
\hline 6,5 & 0,175 & 0,181 & 0,191 & 0,203 & 0,206 & 0,211 & 0,221 & 0,245 & 0,268 & 0,300 & 0,308 & 0,326 & 0,346 \\
\hline
\end{tabular}


T A в д A 1.4 .6$.

Clínker Hornos Lépol S. Blaine $4.500 \mathrm{~cm}^{2} / \mathrm{g}$

Retracción de pasta pura expuesta al aire a temperatura ambiente

\begin{tabular}{cccccccccccccc}
\hline $\begin{array}{c}\text { \% Yeso } \\
\text { añadido }\end{array}$ & $\mathbf{2}$ días & $\mathbf{3}$ días & $\mathbf{4}$ días & $\mathbf{5}$ días & $\mathbf{6}$ días & $\mathbf{7}$ días & $\mathbf{1 4}$ días & $\mathbf{2 1}$ días & $\mathbf{2 8}$ días & $\mathbf{3 5}$ días & $\mathbf{4 2}$ días & $\mathbf{4 9}$ días & $\mathbf{5 6}$ días \\
\hline 1,5 & 0,080 & 0,115 & 0,174 & 0,208 & 0,236 & 0,249 & 0,263 & 0,340 & 0,370 & 0,443 & 0,467 & 0,483 & 0,487 \\
2,0 & 0,061 & 0,107 & 0,138 & 0,171 & 0,199 & 0,212 & 0,215 & 0,312 & 0,341 & 0,396 & 0,424 & 0,445 & 0,447 \\
2,5 & 0,061 & 0,097 & 0,137 & 0,168 & 0,191 & 0,206 & 0,212 & 0,301 & 0,325 & 0,383 & 0,413 & 0,435 & 0,439 \\
3,0 & 0,054 & 0,101 & 0,127 & 0,154 & 0,170 & 0,183 & 0,191 & 0,252 & 0,280 & 0,337 & 0,363 & 0,374 & 0,379 \\
3,5 & 0,059 & 0,089 & 0,110 & 0,132 & 0,147 & 0,159 & 0,178 & 0,228 & 0,254 & 0,298 & 0,323 & 0,341 & 0,343 \\
4,0 & 0,042 & 0,087 & 0,103 & 0,125 & 0,141 & 0,150 & 0,175 & 0,207 & 0,232 & 0,281 & 0,290 & 0,304 & 0,317 \\
4,5 & 0,058 & 0,084 & 0,108 & 0,121 & 0,133 & 0,141 & 0,175 & 0,195 & 0,238 & 0,264 & 0,267 & 0,291 & 0,293 \\
5,0 & 0,066 & 0,090 & 0,117 & 0,127 & 0,136 & 0,154 & 0,177 & 0,209 & 0,256 & 0,276 & 0,285 & 0,310 & 0,314 \\
5,5 & 0,073 & 0,098 & 0,119 & 0,131 & 0,148 & 0,153 & 0,169 & 0,214 & 0,257 & 0,287 & 0,298 & 0,311 & 0,313 \\
6,0 & 0,051 & 0,095 & 0,115 & 0,131 & 0,141 & 0,152 & 0,204 & 0,212 & 0,250 & 0,285 & 0,299 & 0,303 & 0,311 \\
6,5 & 0,085 & 0,113 & 0,138 & 0,155 & 0,159 & 0,174 & 0,227 & 0,246 & 0,261 & 0,312 & 0,328 & 0,329 & 0,344 \\
7,0 & 0,049 & 0,139 & 0,147 & 0,170 & 0,180 & 0,190 & 0,210 & 0,240 & 0,303 & 0,322 & 0,337 & 0,346 & 0,350 \\
\hline
\end{tabular}

\subsection{Comentario parcial}

Al igual que en los hornos Allis-Chalmers, un aumento en el contenido de yeso eleva los valores de las superficies específicas Blaine.

El fraguado inicial, en ocasiones inferior a los $30 \mathrm{~min}$, hace aconsejable que los cementos fabricados con este clínker tengan un $\mathrm{SO}_{3}$ mínimo, superior al $1,7 \%$.

La expansión al autoclave no se ve influenciada por un mayor o menor contenido en yeso; sin embargo la retracción experimentada por las probetas fabricadas con pasta pura, expuestas al ambiente del laboratorio, tienen un mínimo, más o menos amplio, que coincide con las mejores resistencias a corto plazo.

De manera muy similar a los datos alcanzados con el clínker de los hornos Allis-Chalmers, aquí también se detecta la mejora de las resistencias mecánicas a muy corto plazo para determinados márgenes de $\mathrm{SO}_{3}$, el cual va siendo superior a medida que los cementos son más finos.

En los correspondientes a 2.500 y $3.500 \mathrm{~cm}^{2} / \mathrm{g}$ no se ve con demasiada claridad el margen de bondad, en parte por los errores propios de los ensayos; sin embargo, en el de 4.500 $\mathrm{cm}^{2} / \mathrm{g}$, tanto en flexotracción como en compresión, hay un máximo muy claro, correspondiente a un $3,3 \%$ de $\mathrm{SO}_{3}$, a partir del cual, lo mismo en un sentido como en otro, los valores logrados son inferiores.

El porcentaje de retracción mínimo también concuerda con este valor, teniendo una amplitud más estrecha que los de finura más grosera. 

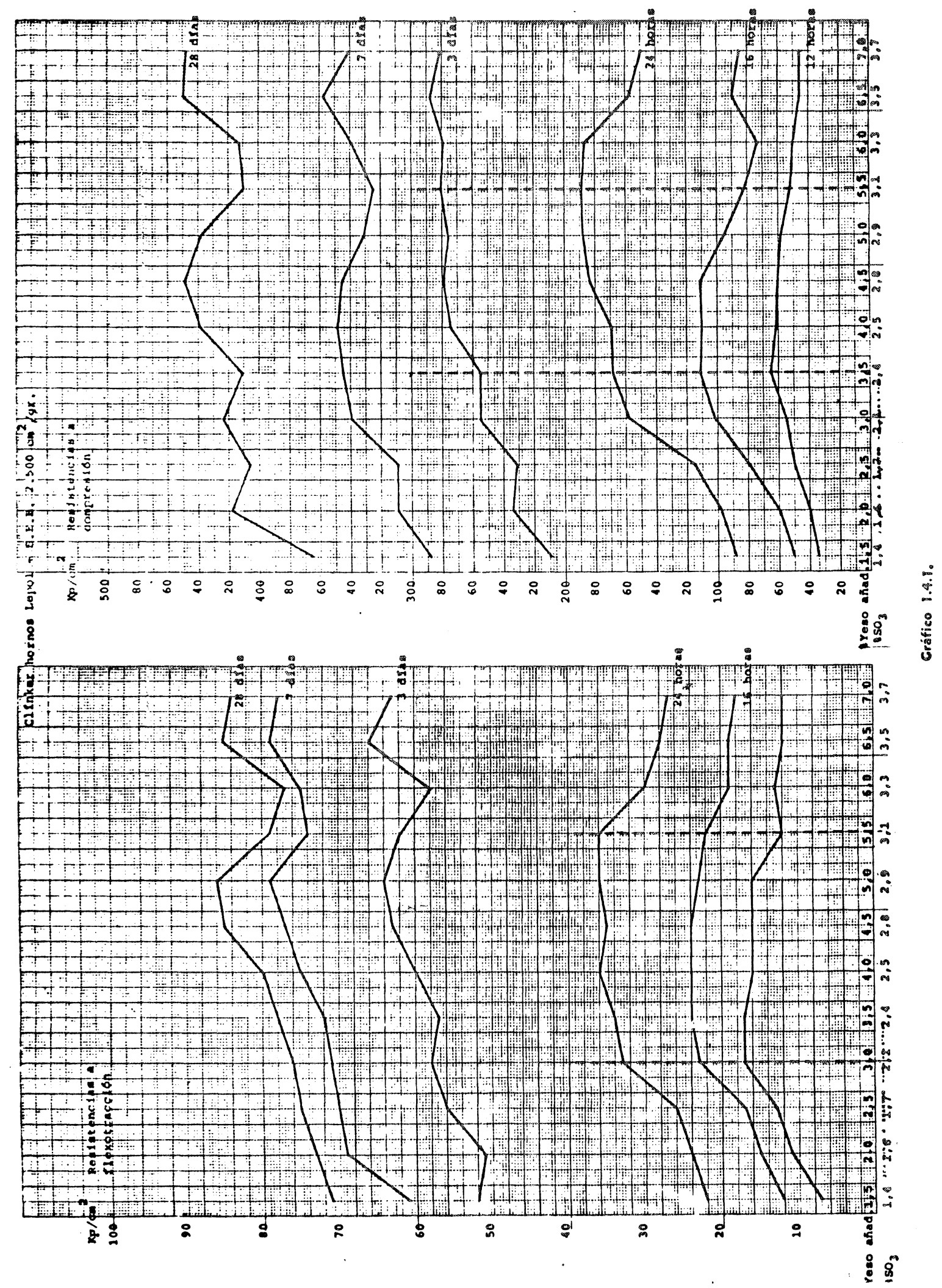


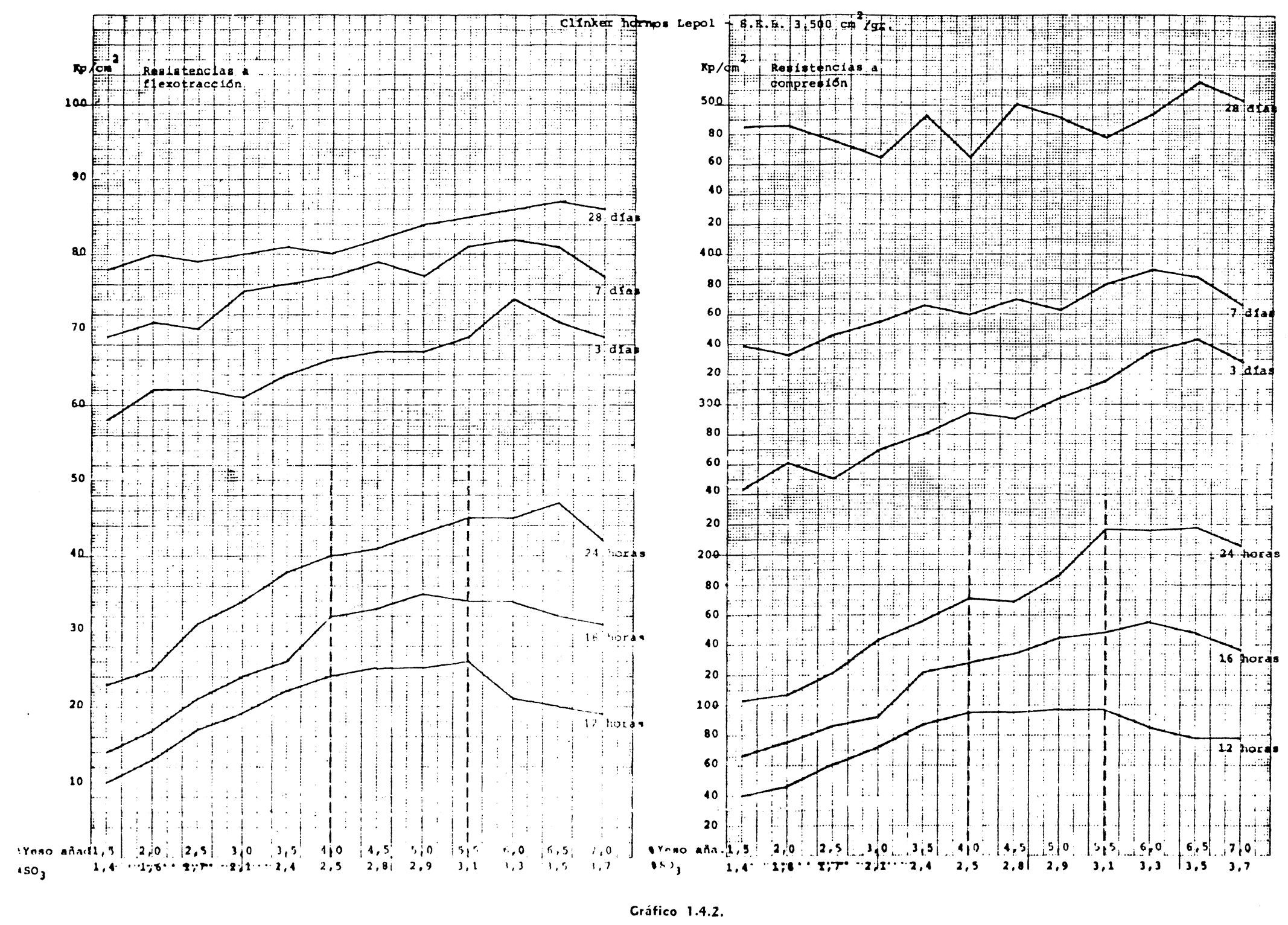



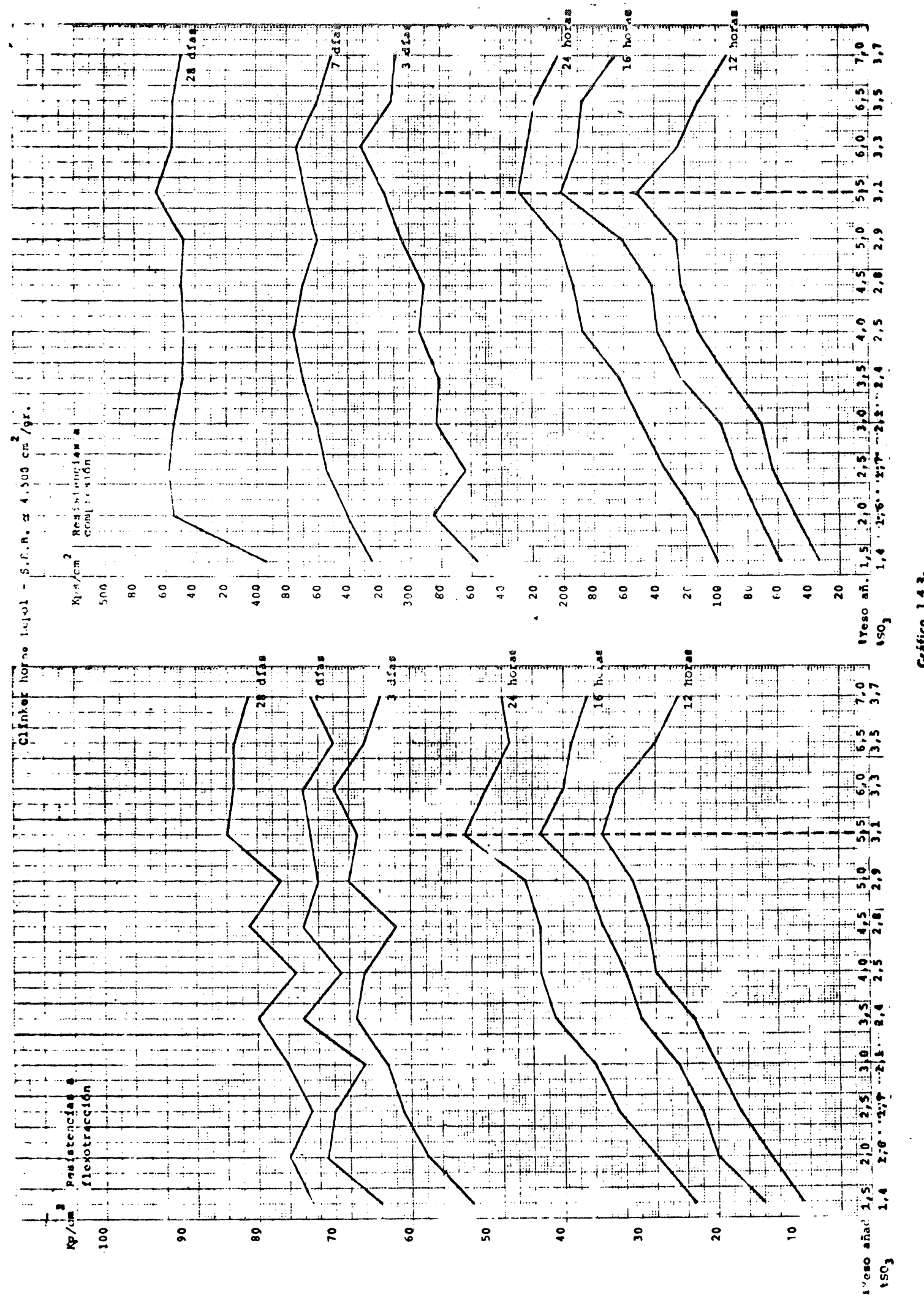

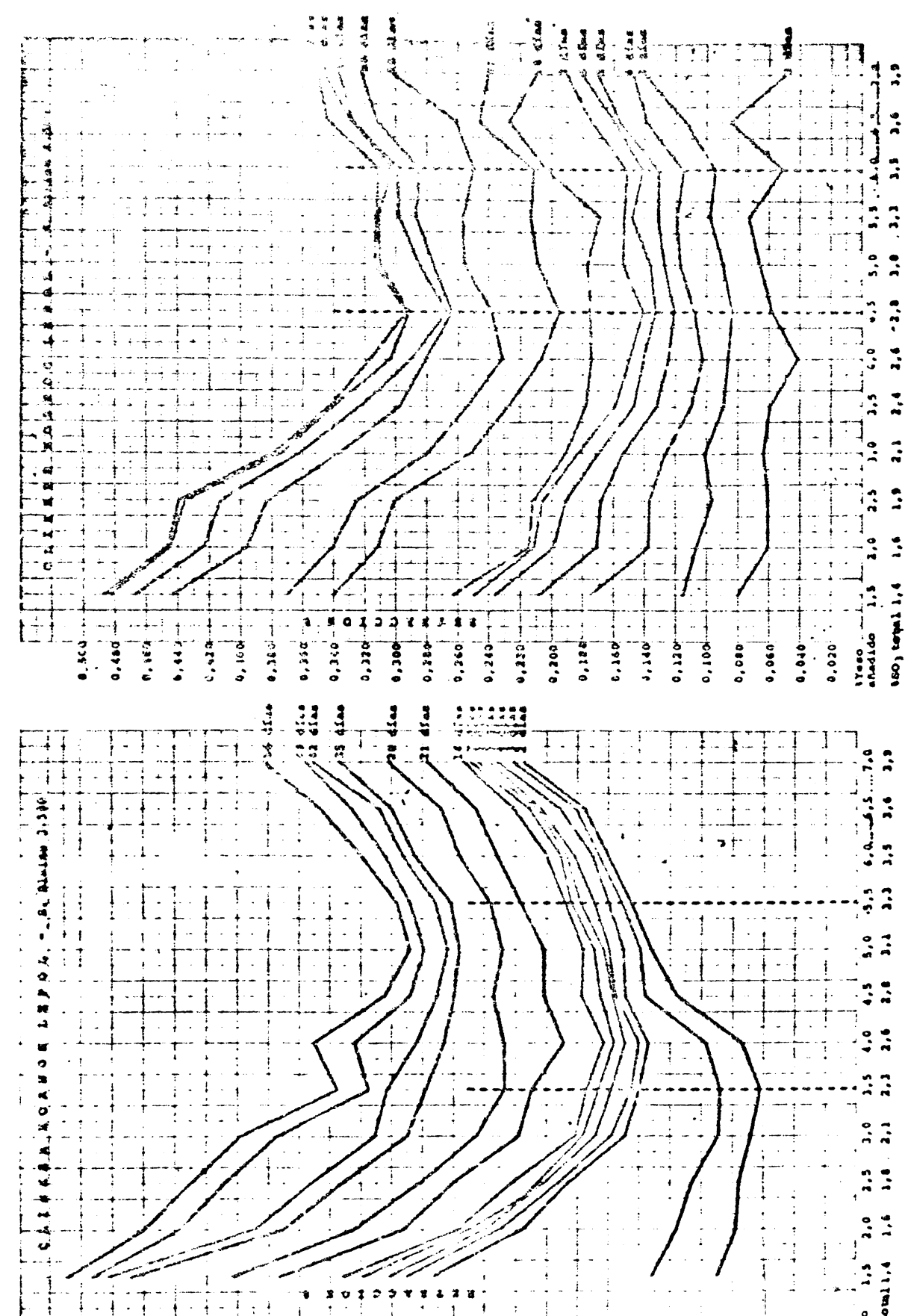

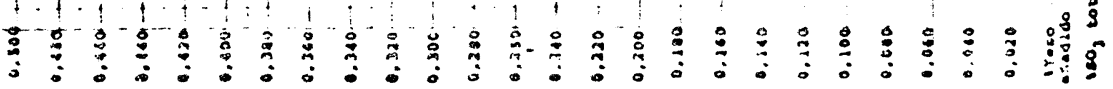

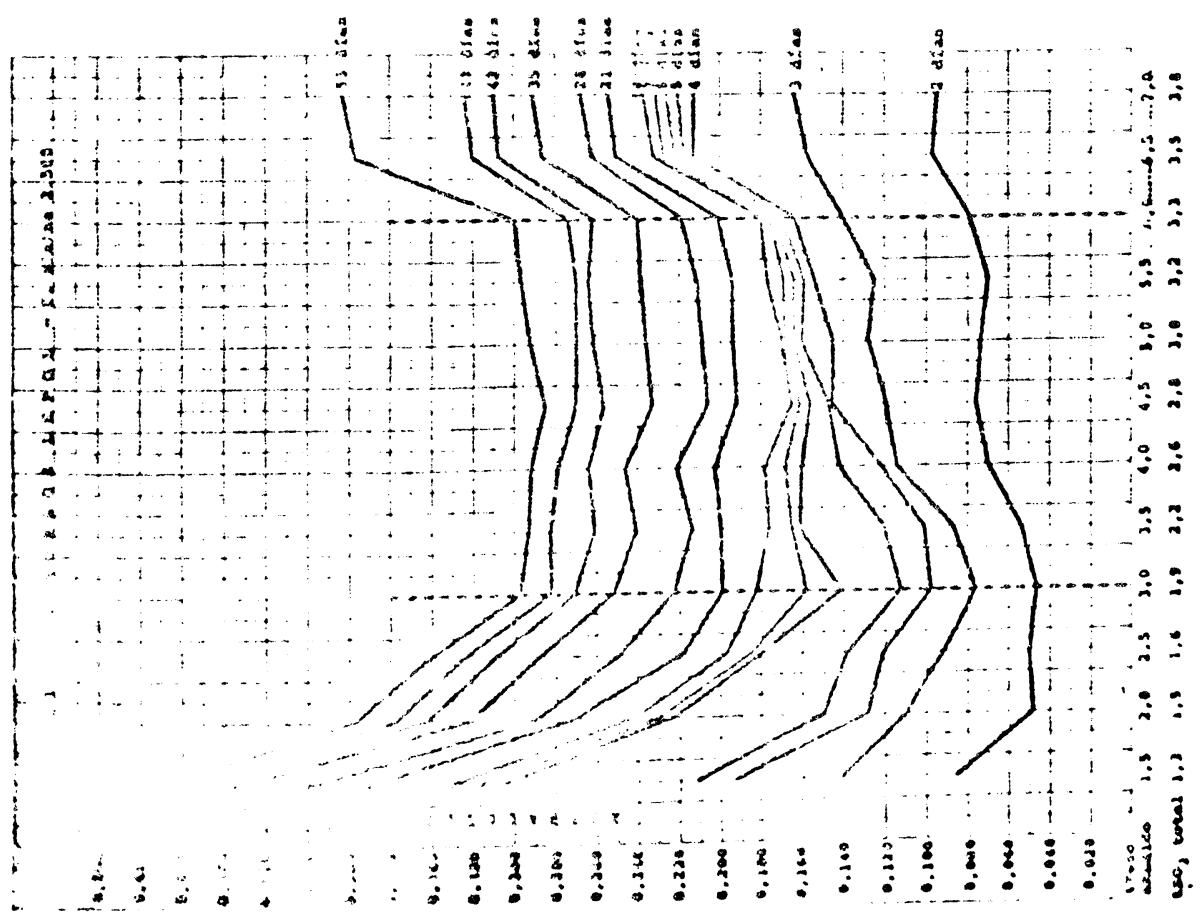




\section{ENSAYO INDUSTRIAL}

\section{ENSAYO INDUSTRIAL BASADO EN LOS DATOS OBTENIDOS EN EL LABORATORIO}

Conocidos los resultados del laboratorio, hemos querido cerciorarnos si efectivamente el cambio del contenido de $\mathrm{SO}_{3}$ tenía una influencia práctica en la molienda industrial.

En una molienda efectuada en la Fábrica n." 3, con un molino Allis-Chalmers modificado (Z) de $420 \mathrm{~kW}$ para obtener P-450 ARI, se controló hora a hora, a lo largo de todo el día, el contenido de $\mathrm{SO}_{3}$, el cual se fue modificando a medida que se tuvo conocimiento del análisis.

Así, en el momento de comenzar la prueba, el $\mathrm{SO}_{3}$ era de $3,2 \%$, del cual se pasó en horas sucesivas a: $3,5,3,6,3,7,4,2$ y 4,4, para volver finalmente al punto de partida: $3,2 \%$.

En la tabla adjunta 2.1. se detallan los valores obtenidos, así como el promedio diario ensayado normalmente para el control de fabricación que nos sirve de comparación con las moliendas parciales efectuadas.

En el gráfico 2.1.1. se representan los valores logrados a flexotracción y compresión y se comparan con los valores obtenidos en el promedio de las 24 horas.

T A B L A 2.1.

Ensayo industrial en un molino "Allis-Chalmers" (Z) P-450 ARI

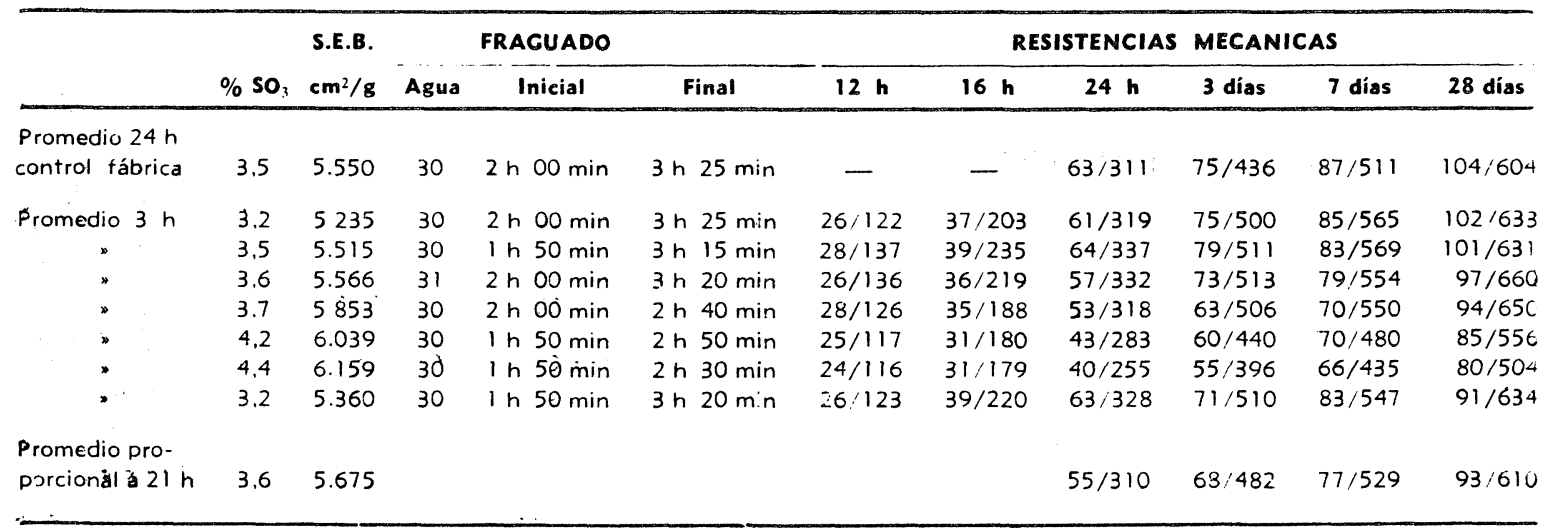

\subsection{Comentario parcial}

Se confirma el aumento de S. E. Blaine con el mayor porcentaje de yeso, llegando en este caso concreto a ser superior a $6.000 \mathrm{~cm}^{2} / \mathrm{g}$ para un $4,4 \%$ de $\mathrm{SO}_{3}$.

Contrariamente a lo que a primera vista pudiera parecer, este elevado Blaine no sóln no origina más altas resistencias, sino que alcanza los valores más bajos a todas las edades.

El valor óptimo está alrededor de $3,5 \%$ de $\mathrm{SO}_{3}$, y a medida que aumenta este porcentaje las resistencias descienden bruscamente hasta un mínimo coincidente con el máximo $\mathrm{SO}_{3}$ antes comentado.

El fraguado parece no tener mucha variación, si bien el final se acorta con porcentajes elevados de yeso.

El promedio de las 24 horas coincide bastante bien con el teórico, proporcional a las 21 horas ensayadas, lo que nos confirma más aún los valores parciales alcanzados. 


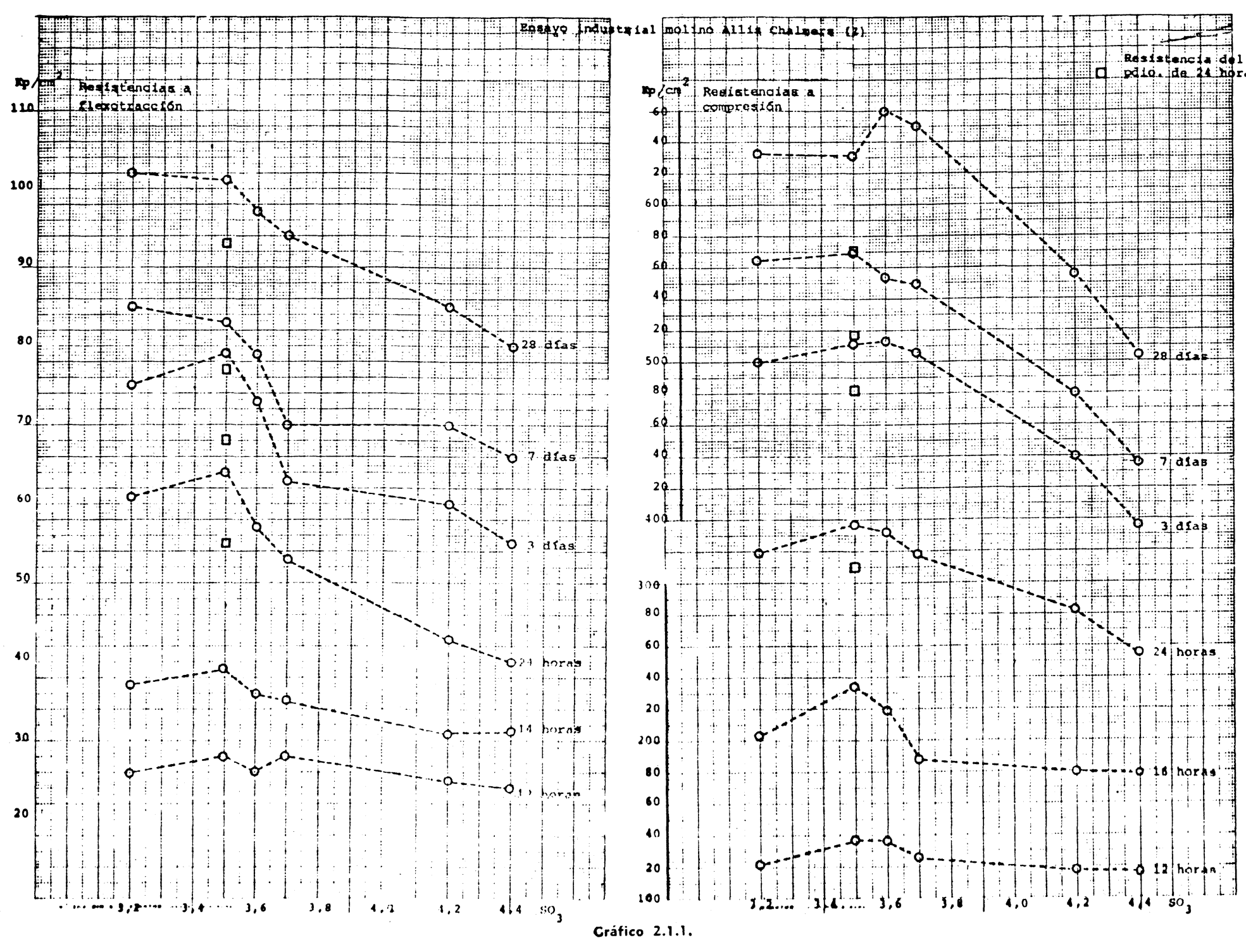




\section{ANALISIS ESTADISTICO}

\section{ANALISIS ESTADISTICO DE LOS CEMENTOS PRODUCIDOS EN LA FABRICA N. 3 DURANTE 21 MESES}

Se han tomado todas las calidades de cemento producido en estos 21 meses, y en el caso deJ P-350 y P-450 se dan también los datos correspondientes a los distintos molinos usados para su fabricación. [Allis-Chalmers, Allis-Chalmers modificado (Z) de $420 \mathrm{~kW}$ y Polysius de $2.300 \mathrm{~kW}$ ].

De cada tipo figuran a continuación en las tablas adjuntas el número de datos por intervalo de $\mathrm{SO}_{3}$, así como las resistencias medias a 24 horas alcanzadas en cada caso.

En los gráficos que acompañamos se representan los datos anteriores y se limitan con trazos verticales los márgenes de $\mathrm{SO}_{3}$, en los cuales se obtienen las máximas resistencias mecánicas a compresión a las 24 horas.

En el caso del P-450 ARI, por considerar que sus resistencias iniciales tienen mayor importancia que en el resto de los cementos, hemos profundizado hasta comprobar las influencias propias del $\mathrm{SO}_{3}$, así como las derivadas de la S.E.B.

\subsection{Valores obtenidos industrialmente en el P-350}

En las tablas y gráficos siguientes se detallan, por tipo de molino, los valores logrados en el P-350:

VARIACION DE LA RESISTENCIA A 24 HORAS CON EL $\mathrm{SO}_{3}$

T A в L A 3.1 .1 .

P-350 molinos Allis-Chalmers

\begin{tabular}{lrrrrrrr}
\hline \multicolumn{1}{c}{ Intervalo \% } & $\mathbf{S O}_{3}$ & $\mathbf{2 , 0 - 2 , 2 9}$ & $\mathbf{2 , 3 - 2 , 5 9}$ & $\mathbf{2 , 6 - 2 . 8 9}$ & $\mathbf{2 , 9 - 3 , 1 9}$ & $\mathbf{3 , 2 - 3 , 4 9}$ & $\mathbf{3 , 5 0 - 3 , 7 9}$ \\
\hline N." de datos por intervalo & 16 & 48 & 45 & 39 & 44 & 12 \\
Porcentaje por intervalo & 8 & 23 & 22 & 19 & 22 & 6 \\
Media por intervalo a $24 \mathrm{~h}\left(\mathrm{kp} / \mathrm{cm}^{2}\right)$ & 122 & 127 & 129 & 117 & 109 & 105 \\
\hline
\end{tabular}

TAB L A 3.1 .2 .

P-350 molinos Polysius

\begin{tabular}{lrrrrrrrr}
\multicolumn{1}{c}{ Intervalo \% } & so $_{3}$ & $\mathbf{2 , 0 - 2 , 2 9}$ & $\mathbf{2 , 3 - 2 , 5 9}$ & $\mathbf{2 , 6 - 2 , 8 9}$ & $\mathbf{2 , 9 - 3 , 1 9}$ & $\mathbf{3 , 2 - 3 , 4 9}$ & $\mathbf{3 , 5 - 3 , 7 9}$ & $\mathbf{3 , 8 - 4 , 0 9}$ \\
\hline $\mathrm{N}$." de datos por intervalo & 5 & 29 & 86 & 111 & 51 & 21 & 6 \\
Porcentaje por intervalo & 2 & 9 & 27 & 37 & 16 & 7 & 2 \\
Media por intervalo a $24 \mathrm{~h}\left(\mathrm{kp} / \mathrm{cm}^{2}\right)$ & 104 & 122 & 113 & 110 & 102 & 99 & 87 \\
\hline
\end{tabular}



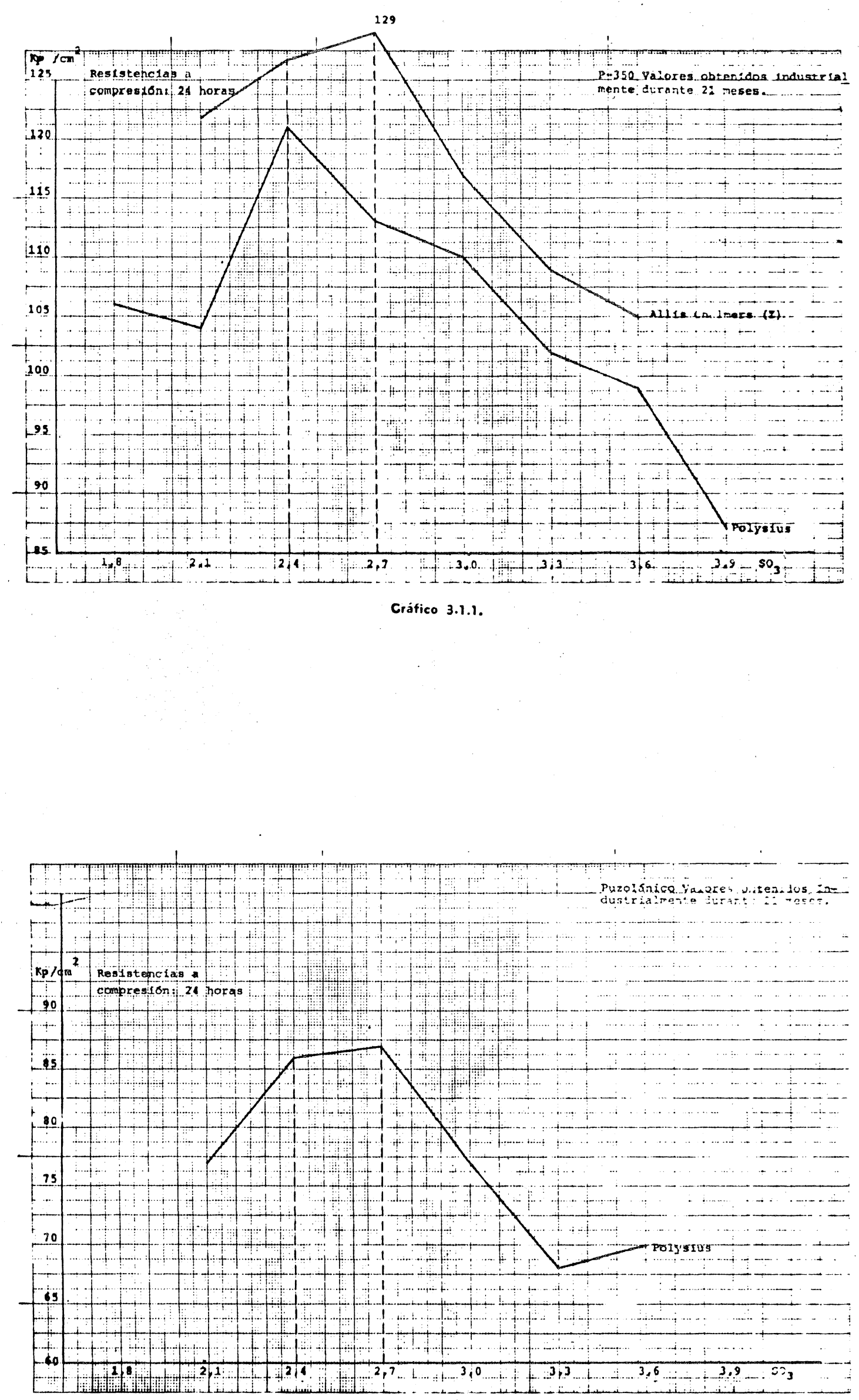

Crático 3.2.1. 


\subsection{Valores obtenidos industrialmente en el Puzolánico}

En la ta.bla y gráfico 3.2.1. se exponen los resultados obtenidos en el cemento puzolánics con porcentajes de $\mathrm{SO}_{3}$ que van de 2,0 a 3,8 :

T A B L A 3.2 .1 .

Cemento puzolánico molinos Polysius

\begin{tabular}{lrrrrrr}
\hline \multicolumn{1}{c}{ Intervalo \% so $_{3}$} & $\mathbf{2 , 0 - 2 , 2 9}$ & $\mathbf{2 , 3 - 2 , 5 9}$ & $\mathbf{2 , 6 - 2 , 8 9}$ & $\mathbf{2 , 9 - 3 , 1 9}$ & $\mathbf{3 , 2 - 3 , 4 9}$ & $\mathbf{3 , 5 - 3 , 7 9}$ \\
\hline N." de datos por intervalo & 7 & 12 & 67 & 64 & 35 & 8 \\
Porcentaje por intervalo & 4 & 6 & 35 & 33 & 18 & 4 \\
Media por intervalo a $24 \mathrm{~h}\left(\mathrm{kp} / \mathrm{cm}^{2}\right)$ & 77 & 86 & 87 & 77 & 68 & 70 \\
\hline
\end{tabular}

\subsection{Valores obtenidos industrialmente en el P-450}

Al igual que en el P-350 en las tablas 3.3.1. y 3.3.2., presentamos los resultados alcanzado: para la calidad P-450 separándolos por el tipo de molino utilizado en su fabricación.

En el gráfico 3.3.1. figuran estos datos:

T A B L A 3.3 .1$.

Cemento P-450 Allis-Chalmers (Z)

\begin{tabular}{lrrrrrrr}
\hline \multicolumn{1}{c}{ Intervalo \% so, } & $\mathbf{2 , 2 - 2 , 5 9}$ & $\mathbf{2 , 6 - 2 , 8 9}$ & $\mathbf{2 , 9 - 3 , 1 9}$ & $\mathbf{3 , 2 - 3 , 4 9}$ & $\mathbf{3 , 5 - 3 , 7 9}$ & $\mathbf{3 , 8 - 4 , 0 9}$ \\
\hline N." de datos por intervalo & 6 & 18 & 22 & 41 & 29 & 24 \\
Porcentaje por intervalo & 4 & 13 & 16 & 29 & 21 & 17 \\
Media por iniervalo a $24 \mathrm{~h}\left(\mathrm{kp} / \mathrm{cm}^{2}\right)$ & 164 & 174 & 176 & 180 & 162 & 145 \\
\hline
\end{tabular}

T A B L A 3.3 .2 .

Cemento P-450 Polysius

\begin{tabular}{|c|c|c|c|c|c|}
\hline Intervalo $\% \mathrm{SO}_{3}$ & $2,0-2,29$ & $2,3-2,59$ & $2,6-2,89$ & $2,9-3,19$ & $3,2-3,49$ \\
\hline Porcentaje por intervalo & 8 & 20 & 37 & 24 & 11 \\
\hline
\end{tabular}



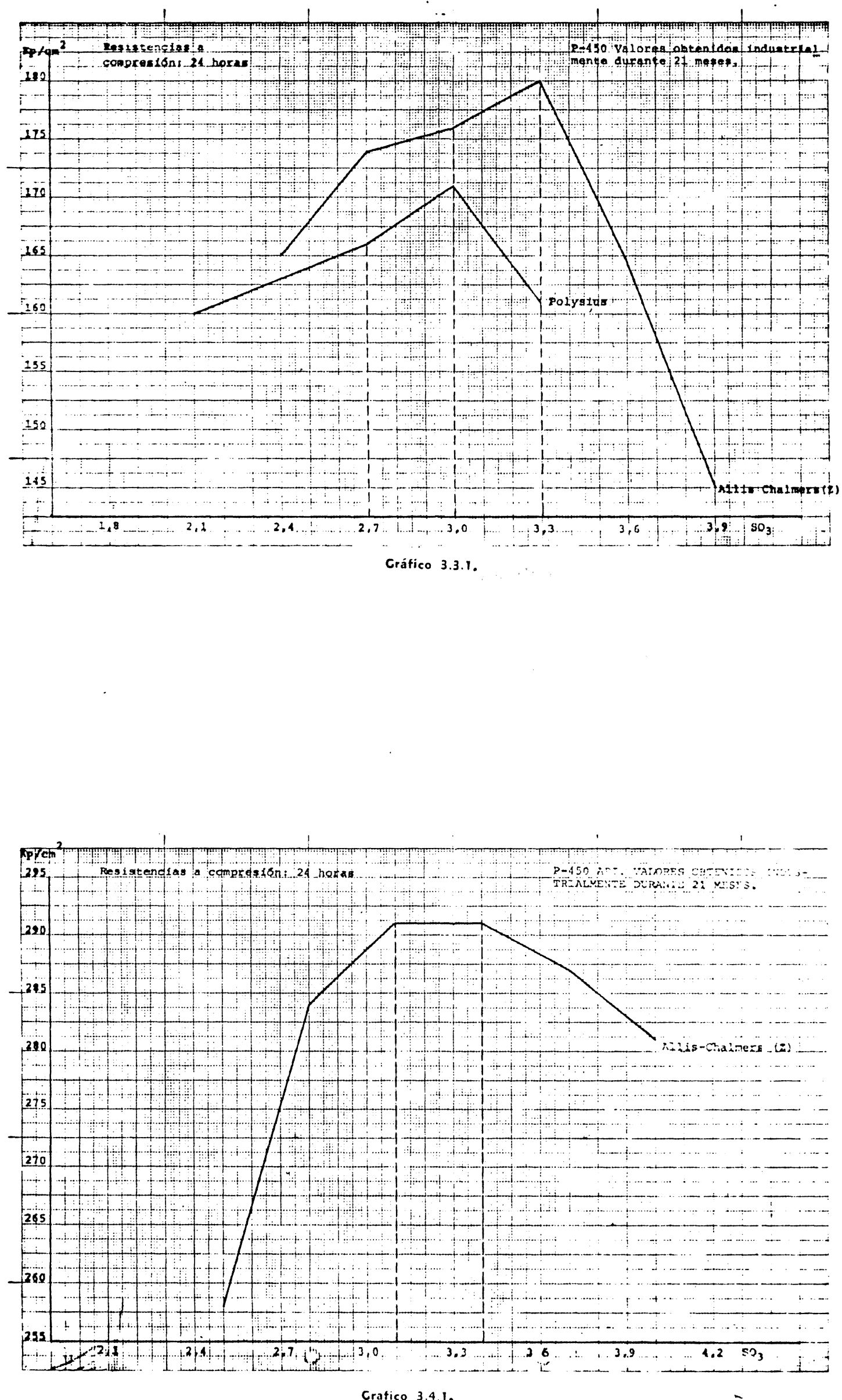


\subsection{Valores obtenidos industrialmente en el P-450 ARI}

En la tabla 3.4.1. se exponen los datos correspondientes al P-450 ARI, y en el gráfico 3.4.1. se representan dichos valores logrados en 21 meses de fabricación:

T A B L A 3.4 .1$.

Cemento P-450 ARI Allis-Chalmers (Z)

\begin{tabular}{|c|c|c|c|c|c|c|}
\hline Intervalo $\%$ SO $_{3}$ & $2,4-2,69$ & $2,7-2,99$ & $3,0-3,29$ & $3,3-3,59$ & $3,6-3,89$ & $3,9-4,19$ \\
\hline Porcentaje por intevalo & 5 & 24 & 31 & 18 & 15 & 7 \\
\hline Media por intevalo a $\check{~} 4 \mathrm{~h}\left(\mathrm{kp} / \mathrm{cm}^{2}\right)$ & 258 & 284 & 291 & 291 & 287 & 281 \\
\hline
\end{tabular}

Para profundizar más en el exacto valor de estos datos debemos, en primer lugar, saber si siguen una curva gaussiana y, para ello, hemos hecho uso del ensayo de PEARSON, el cual exige para que una curva resulte normal se cumpla que

$$
x^{2}=\frac{\sum\left(N_{\mathrm{i}}-N P_{\mathrm{i}}\right)^{2}}{N P_{\mathrm{i}}}
$$

sea menor al $X^{2}$ teórico determinado por PEARSON (ver tabla de PEARSON), siendo $N_{\mathrm{i}}$ la frecuencia observada; $P_{\mathrm{i}}$ la probabilidad teórica correspondiente a la ley de LAPLACE-GAUSS y $N$ el total de observaciones.

\begin{tabular}{lcccccccc}
\hline \multicolumn{1}{c}{$X^{2}$ de Pearson } \\
\hline Número de grados de libertad & $\mathbf{1}$ & $\mathbf{2}$ & $\mathbf{3}$ & $\mathbf{4}$ & $\mathbf{5}$ & $\mathbf{6}$ & $\mathbf{7}$ \\
\hline Valor de $X^{\mathbf{2}}$ (umbral de 0,05$)$ & 3,84 & 5,99 & 7,81 & $\mathbf{9 , 4 9}$ & 11,07 & 12,59 & 14,07 \\
Número de grados de libertad & 8 & 9 & 10 & 15 & 20 & 25 & 30 \\
Valor de $X^{2}$ (umbral de 0,05 ) & 15,51 & 16,92 & 18,31 & 25,00 & 31,41 & 37,65 & 43,77 \\
\hline
\end{tabular}

T А в L A 3.4 .2 .

\begin{tabular}{|c|c|c|c|c|c|c|c|}
\hline $\begin{array}{l}\text { Intervalo } \\
\left(\mathbf{k p} / \mathrm{cm}^{2}\right)\end{array}$ & $\begin{array}{c}\text { Valores } \\
\text { centrales } \\
x_{i}\end{array}$ & $\begin{array}{c}\text { Número de } \\
\text { observaciones } \\
N_{\mathrm{i}} \\
\end{array}$ & $\begin{array}{c}\text { Porcentaje } \\
\text { intervalo }\end{array}$ & $z=\left(x_{i}-\bar{x}\right) / s$ & $\begin{array}{c}\text { Probabilidades } \\
\text { parciales } \\
\text { teóricas } \\
\mathbf{F}(\mathbf{Z}) \\
\end{array}$ & 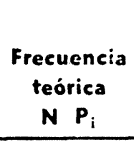 & $x$ \\
\hline \multirow[t]{2}{*}{$\begin{array}{l}200-219,9 \\
220-239,9 \\
240-259,9 \\
260-279,9 \\
280-299,9 \\
300-319,9 \\
320-339,9\end{array}$} & $\begin{array}{l}210 \\
230 \\
250 \\
270 \\
290 \\
310 \\
330\end{array}$ & $\begin{array}{c}3 \\
10 \\
24 \\
60 \\
79 \\
68 \\
21\end{array}$ & $\begin{array}{r}1 \\
4 \\
9 \\
22 \\
30 \\
26 \\
8\end{array}$ & $\begin{array}{l}3,07 \\
2,26 \\
1,45 \\
0,65 \\
0,16 \\
0,97 \\
1,78\end{array}$ & $\begin{array}{l}0,006 \\
0,033 \\
0,140 \\
0,317 \\
0,394 \\
0,249 \\
0,085\end{array}$ & $\begin{array}{r}1,28 \\
7,07 \\
30,03 \\
67,99 \\
84,51 \\
53,41 \\
18,23\end{array}$ & $\begin{array}{l}2,3 \\
1,2 \\
1,2 \\
0,9 \\
0,3 \\
2,9 \\
0,4\end{array}$ \\
\hline & & $N=265$ & & & & & $x^{2}=9,2$ \\
\hline
\end{tabular}

$\bar{X}=286,2 \mathrm{kp} / \mathrm{cm}^{2} \quad e=20 \mathrm{kp} / \mathrm{cm}^{2}$

$S=24,7 \mathrm{kp} / \mathrm{cm}^{2}$

N.? grados de libertad $=7-3=4 \quad X^{2}$ (PEARSON) $=9,5$

$X^{2}$ (Calculado) $=9.2-9,5=X^{2}$ (teórico de Pearson) 
De donde deducimos que, efectivamente, los datos con los que trabajamos están agrupados según una curva de GAUSS.

Una vez aclarado este punto trataremos de comprobar por el ensayo de FISHER-SNEDECOR si las resistencias medias por intervalo a 24 horas se hallan efectivamente influenciadas por el porcentaje de $\mathrm{SO}_{3}$, o bien si las diferencias encontradas se deben al azar o a otros factores no estudiados aquí:

Tabla de distribución del "F" de FISHER-SNEDECOR

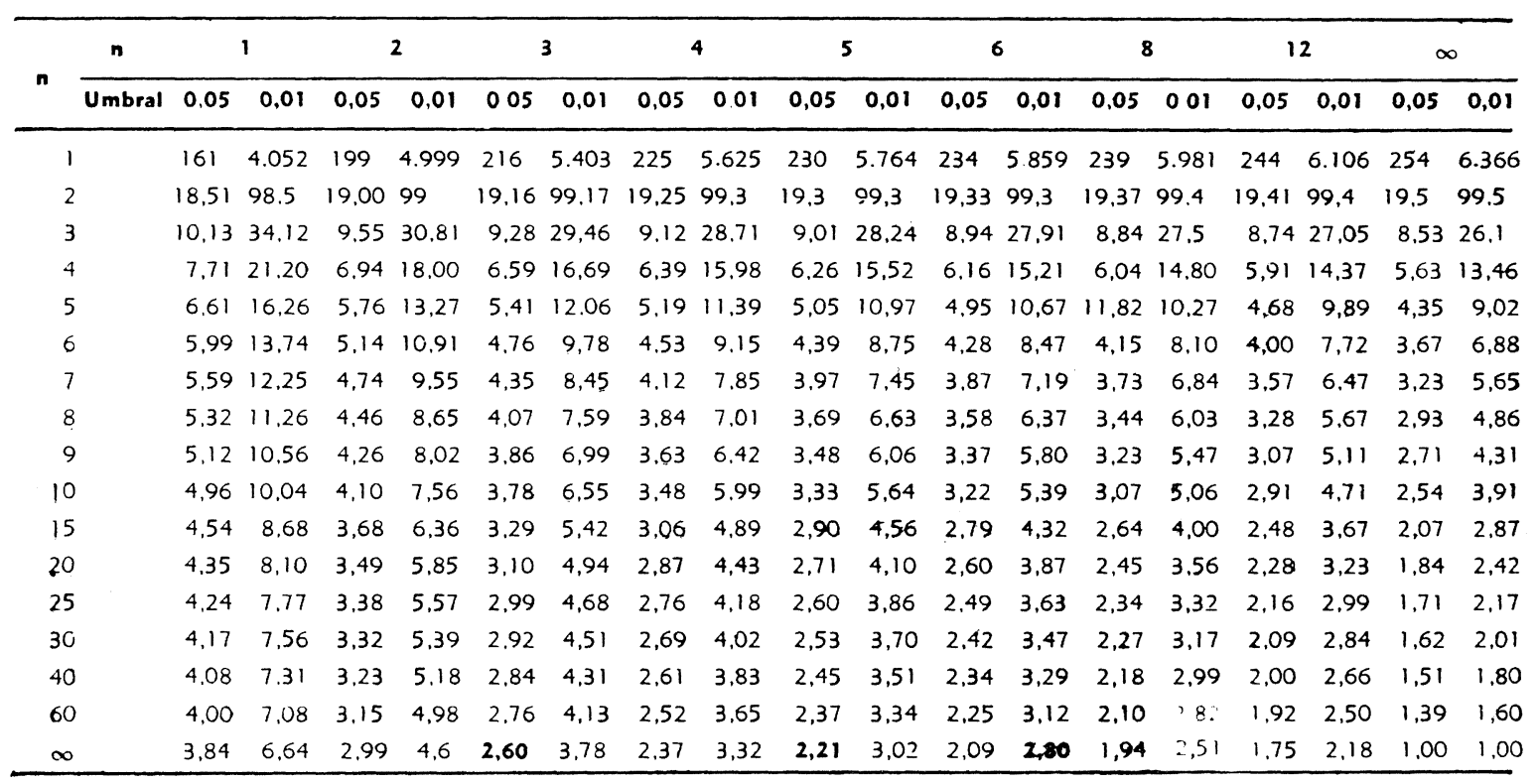

En la tabla adjunta detallamos los pasos seguidos para la aclaración de este punto:

T A B L A 3.4 .3 .

\begin{tabular}{|c|c|c|c|c|c|c|}
\hline $\begin{array}{c}\text { Intervalo } \\
\% \text { so } \\
\end{array}$ & $\begin{array}{l}\text { N.o datos } \\
\text { intervalo }\end{array}$ & $\begin{array}{l}\text { Porcentaje } \\
\text { intervalo }\end{array}$ & $\begin{array}{c}\begin{array}{c}\text { Media } \\
\text { por } \\
\text { intervalo }\end{array} \\
\end{array}$ & $\left.\overline{\left(x_{i}\right.}-\bar{x}\right)^{2}$ & $N_{i}\left(\bar{x}_{i}-\bar{x}\right)^{2}$ & $\left(x_{i j}-\bar{x}_{i}\right)^{2}$ \\
\hline $2,40-2,69$ & 13 & 5 & 258 & 795,2 & $10.337,6$ & 10.184 \\
\hline $2,70-2,99$ & 64 & 24 & 284 & 4,8 & 307,2 & 32.123 \\
\hline $3,00-3,29$ & 81 & 31 & 291 & 27,0 & $2.187,0$ & 50.697 \\
\hline $3,30-3,59$ & 49 & 18 & 291 & 27,0 & $1.3<3,0$ & 44.829 \\
\hline $3,60-3,89$ & 39 & 15 & 287 & 1,4 & 54,6 & 15.754 \\
\hline \multirow[t]{2}{*}{$3,90 \cdot 4,19$} & 19 & 7 & 281 & 27,0 & 513,0 & 12.870 \\
\hline & $N=265$ & & & & $14.722,4$ & 166.457 \\
\hline
\end{tabular}

$$
\overline{\bar{X}}=286,2 \quad s^{2}=\frac{14.722,4}{6-1}=2.944 .5 \quad S^{2}=\frac{166.457}{265-6}=642,7
$$$$
F^{\prime}=\frac{s^{2}}{S^{2}}=\frac{2.944,5}{642,7}=4,58 \quad \text { "F" }=2,21 \text { (Tabla de distribución }
$$
de Fisher-Snedecor)

$$
F^{\prime}=4,58>2,21=\text { " } F " \text { (Fisher Snedecor) }(0,05)
$$


Lo cual es lo mismo que decir que los intervalos de $\mathrm{SO}_{3}$ antes mencionados ejercen una influencia profunda en la obtención de resistencias mecánicas a 24 horas en el P-450 ARI, existiendo un $95 \%$ de posibilidades de que estas conclusiones sean ciertas.

De idéntica forma tratamos de comprobar la influencia de la S. E. Blaine en las resistencias a 24 horas del P-450 ARI y, por ello, hemos agrupado de nuevo los datos obtenidos industrialmente a lo largo de los 21 meses estudiados, en intervalos de $300 \mathrm{~cm}^{2} / \mathrm{g}$.

Los datos se detallan en la siguiente tabla:

TABLA 3.4 .4 .

\begin{tabular}{|c|c|c|c|c|c|c|}
\hline $\begin{array}{c}\text { Intervalo } \\
\text { S.E.B. }\end{array}$ & $\begin{array}{c}\mathbf{N} . " \text { datos } \\
\text { intervalo } \\
\mathbf{N}_{\mathrm{i}}\end{array}$ & $\begin{array}{c}\text { Porcentaje } \\
\text { intervalo }\end{array}$ & $\begin{array}{c}\text { Media } \\
\text { por } \\
\text { intervalo }\end{array}$ & $\left(\bar{x}{ }_{i}-\bar{x}\right)^{2}$ & $N_{i}\left(\bar{x}_{i}-\bar{x}\right)^{2}$ & $\left(x_{i j}-\overline{x_{i}}\right)^{2}$ \\
\hline $3.900-4.199$ & 13 & 4,9 & 268 & 338,6 & $4.401,8$ & 12.940 \\
\hline $4.200-4.499$ & 15 & 5,7 & 277 & 88,4 & $1.326,0$ & 12.302 \\
\hline $4.500-4.799$ & 40 & 15,2 & 279 & 54,8 & $2.192,0$ & 33.189 \\
\hline $4.800-5.099$ & 67 & 25,5 & 288 & 2,6 & 174,2 & 38.349 \\
\hline $5.100-5.399$ & 89 & 33,9 & 288 & 2,6 & 231,4 & 45029 \\
\hline $5.400-5.699$ & 31 & 11,8 & 296 & $92, z$ & $2.858,2$ & 9.250 \\
\hline \multirow[t]{2}{*}{$5.700-6.000$} & 8 & 3,0 & 301 & 213,2 & $1.705,6$ & $1.74 t$ \\
\hline & $N=263$ & & & & $12.889,2$ & 152.805 \\
\hline
\end{tabular}

$$
X=286,4 \quad s^{2}=\frac{12.889,2}{7-1}=2.148,2 \quad S^{2}=\frac{152.805}{263-7}=596,8
$$

$F^{\prime}=\frac{s^{2}}{S^{2}}=\frac{2.148,2}{596,8}=3.60$

$$
F^{\prime}=3,60>2,80=\text { "F" (Fisher-Sn€decor) }(0,01)
$$

Lo cual, vista la amplitud tan grande existente en la S.E.B. (3.900-6.000), no es extraño y nos confirma la tesis por todos sabida de la influencia que ejerce la S. E. Blaine en las resistencias.

No obstante, se observa que no es normal la fabricación de P-450 ARI con S. E. Blaine de 3.900 a 4.500 , por un lado, y las superiores a 5.700 , por otro, como lo demuestra que el $87 \%$ de las muestras se encuentre entre 4.500 y $5.700 \mathrm{~cm}^{2} / \mathrm{g}$.

Tomando esta fracción como característica del cemento fabricado en la Fábrica n." 3 para la calidad P-450 ARI, a ella nos referiremos para conocer la verdadera influencia de la superficie específica en las resistencias a corto plazo en el campo en que suele moverse para este tipo de cemento: 
T A B L A 3.4 .5 .

\begin{tabular}{|c|c|c|c|c|c|c|}
\hline $\begin{array}{l}\text { Intervalo } \\
\text { S.E.B. }\end{array}$ & $\begin{array}{c}N_{0}^{0} \text { datos } \\
\text { intervalo } \\
\mathbf{N}_{\mathrm{i}}\end{array}$ & $\begin{array}{l}\text { Porcentaje } \\
\text { intervalo }\end{array}$ & $\begin{array}{c}\text { Media } \\
\text { por } \\
\text { intervalo }\end{array}$ & $\left.\overline{\left(x_{i}\right.}-\bar{x}\right)^{2}$ & $N_{i}\left(\bar{x}_{i}-\bar{x}\right)^{2}$ & $\left(\mathbf{x}_{i},-\overline{\mathbf{x}}_{\mathrm{i}}\right)^{2}$ \\
\hline $4.500-4.799$ & 40 & 17,6 & 279 & 72,25 & $2.890,00$ & 33.189 \\
\hline $4.800-5.099$ & 67 & 29,5 & 288 & 0,25 & 16,75 & 38.349 \\
\hline $5.100-5.399$ & 89 & 39,2 & 288 & 0,25 & $22,<5$ & 45.029 \\
\hline \multirow[t]{2}{*}{$5.400-5.700$} & 31 & 13,7 & 296 & 72,25 & $2.239,75$ & 9.250 \\
\hline & $N=227$ & & & & $5.168,75$ & 125.817 \\
\hline
\end{tabular}

$\bar{X}=287,5 \quad s^{2}=\frac{5.168 .75}{4-1}=1.722,9 \quad S^{2}=\frac{125.817}{227-4}=564,2$

$F^{\prime}=\frac{s^{2}}{S^{2}}=\frac{1.722,9}{564,2}=3,05$

$F^{\prime}=13,05>2,60=$ "F" (Fisher-Snedecor) $(0,05)$

Esto es. para intervalos de $300 \mathrm{~cm}^{2} / \mathrm{g}$, en los límites arriba citados. la S.E.B. influye en las resistencias a 24 horas del P-450 ARI, con un grado de probabilidad de que esto sea cierto, idéntico al alcanzado con la relación $\mathrm{SO}_{3} / 24$ horas.

\subsection{Conclusiones parciales}

Existe una buena concordancia entre los datos obtenidos en el laboratorio y los obtenidos estadísticamente de los controles de fabricación, como se demuestra en la siguiente tabla:

T A в L A 3.5 .1$.

Valores óptimos de $\mathrm{SO}_{3}$

\begin{tabular}{|c|c|c|c|c|}
\hline \multirow[t]{2}{*}{ Călidad } & \multirow{2}{*}{$\begin{array}{r}\text { Resultados } \\
\text { Hornos }\end{array}$} & \multirow{2}{*}{$\begin{array}{l}\text { Obtenidos en el } \\
\text { Allis-Chalmers }\end{array}$} & \multirow{2}{*}{$\begin{array}{l}\text { laboratorio } \\
\text { Hornos Lépol }\end{array}$} & \multirow{2}{*}{$\begin{array}{l}\text { Resultados estadisticos } \\
\text { de moliendas industriales } \\
\text { (21 meses) }\end{array}$} \\
\hline & & & & \\
\hline $2.500 \mathrm{~cm}^{2} / \mathrm{g}$ & & $8-3,1$ & $2,<-3,2$ & - \\
\hline P-350 Allis-Chalmers & & - & - & $2,4-2,7$ \\
\hline P-350 Polysius & & - & - & $2,4-2,7$ \\
\hline Puzolánico Polysius & & - & - & $2,4-2,7$ \\
\hline $3.500 \mathrm{~cm}^{2} / \mathrm{g}$ & & $6-3,3$ & $2,6-3,3$ & - \\
\hline P-450-Allis-Chalmers (Z) & & - & -- & $3,0-3,3$ \\
\hline P-450 Polysius & & - & - & $2,7-3,0$ \\
\hline $4.500 \mathrm{~cm}^{2} / \mathrm{g}$ & & $3-3,6$ & 3,3 & - \\
\hline P-450 ARt Allis-Chalmers (Z) & & -- & -. & $3,1-3,4$ \\
\hline
\end{tabular}


El ensayo de FISHER-SNEDECOR nos confirma la validez de la influencia del $\mathrm{SO}_{3}$ en las resistencias iniciales, así como la S.E.B. en intervalos de $300 \mathrm{~cm}^{2} / \mathrm{g}$, aunque en límites estrechos esta variación sea mínima, como lo demuestra que cerca del $60 \%$ de las muestras estudiadas, con superficies que van desde 4.800 a 5.400 , presenten idéntica resistencia media de $288 \mathrm{kp} / \mathrm{cm}^{2}$, al plazo de 24 horas.

Sin embargo, para márgenes más amplios, su influencia es considerable, como se observa en la tabla 3.4.4.

En todas las calidades queda bien patente que a ambos lados de la zona "crítica" las resistencias disminuyen, siendo, en ocasiones, este descenso mucho más brusco, con porcentajes altos en $\mathrm{SO}_{3}$, cercanos al $4 \%$.

\section{CONCLUSIONES GENERALES}

$1^{a} \quad$ Del presente estudio se deduce que cada tipo de cemento, molido a diferente superficie específica, tiene un contenido óptimo de adición de yeso para la obtención de las máximas resistencias mecánicas iniciales.

2. ${ }^{2}$ Para los tipos de cemento estudiados, las resistencias iniciales más altas, así como las retracciones mínimas medidas en la pasta pura, se obtienen en unas zonas óptimas de $\mathrm{SO}_{3}$ total comprendidas entre:

2,4 a $2,7 \%$ (P-350 y Puzolánico).

2,9 a $3,3 \% \quad(\mathrm{P}-450)$.

3,1 a $3,4 \%$ (P-450 ARI).

Naturalmente, los extremos de estas zonas serán los más adecuados para épocas de frío y calor.

3. ${ }^{a} \quad$ Existe un mínimo de $\mathrm{SO}_{3}$, cercano al $1,7 \%$, al cual por ningún motivo hay que llegar, a menos de correr el riesgo de fabricar cemento rápido.

\section{B I B L I O G R A F I A}

(1) Bogue, R. H.: La química del cemento portland. 1952.

(2) TAYloR, H. F. W.: The chemistry of cements. 1964.

(3) Fritz KeIL.: Cemento. 1973.

(4) Tenoutasse, N.: Influence du gypse sur l'hydratation de la phase ferrite des ciments portland. Revue des Niatériaux de Construction núms. 604 y 614. 
(5) Joisel, A.: Prise et fausse-prise des ciments. Revue des Matériaux de Construction, núm. 649.

(6) Jolibois, P. et Nicol, A.: L'influence de la presence du sulfate de calcium sur la prise et la résistence des ciments silicates. Extrait de la Revue des Matériaux de Construction. Publication technique número 4. Cerilh.

(7) JASPERS, M. J. M.: Recherche, controle et statistique en cimenterie. Revue des Matériaux de Construction, núm. 624.

(8) Locher, F. W.: Erstarren und anfangsfestigkeit von zement. Zement-Kalk-Gips, febrero 1973.

(9) Schwiete, H. E. y Ludwig, J.: Sobre el empleo de distintos compuestos sulfatados en la fabricación de cemento. IV Asamblea general de la eurogypsum. Madrid 1964.

(10) Calleja, J.: Puntos de vista sobre el contenido de yeso de los cementos portland. Ultimos Avances 611-67, núm. 120, págs. 41-49. 1965.

(11) Margarit, A. y Puig, M.: Influencia de la presencia del sulfato cálcico sobre el fraguado del cemento. Monografía núm. 220, IETcc. 1962.

(12) Budnikov, P. P.: Role of gypsum in the hardening of hidraulic cements. Paper IV Sl. IV International symposium on the chemistry of cement. Washington. 1960.

(13) Copeland, L. E. y Kantro, D. L.: Hydration of portland cement. V International symposium on the chemistry of cement. Tokio. 1968.

(14) Locher, F. W. y Richartz, W.: Study on the hydration mechanism of cement. The VI International congress on the chemistry of cement. Moscou. 1974.

(15) Lerch, W.: The Influence of gypsum on the hidration and properties of portland cement pastes. A. M. Soc. testing mater., proc. $46,1.252-1.292$ (1946). 